\title{
Clusters versus GPUs for Parallel Target and Anomaly Detection in Hyperspectral Images
}

\author{
Abel Paz and Antonio Plaza \\ Department of Technology of Computers and Communications, University of Extremadura, 10071 Caceres, Spain \\ Correspondence should be addressed to Antonio Plaza, aplaza@unex.es
}

Received 2 December 2009; Revised 18 February 2010; Accepted 19 February 2010

Academic Editor: Yingzi Du

Copyright () 2010 A. Paz and A. Plaza. This is an open access article distributed under the Creative Commons Attribution License, which permits unrestricted use, distribution, and reproduction in any medium, provided the original work is properly cited.

Remotely sensed hyperspectral sensors provide image data containing rich information in both the spatial and the spectral domain, and this information can be used to address detection tasks in many applications. In many surveillance applications, the size of the objects (targets) searched for constitutes a very small fraction of the total search area and the spectral signatures associated to the targets are generally different from those of the background, hence the targets can be seen as anomalies. In hyperspectral imaging, many algorithms have been proposed for automatic target and anomaly detection. Given the dimensionality of hyperspectral scenes, these techniques can be time-consuming and difficult to apply in applications requiring real-time performance. In this paper, we develop several new parallel implementations of automatic target and anomaly detection algorithms. The proposed parallel algorithms are quantitatively evaluated using hyperspectral data collected by the NASA's Airborne Visible Infra-Red Imaging Spectrometer (AVIRIS) system over theWorld Trade Center (WTC) in New York, five days after the terrorist attacks that collapsed the two main towers in theWTC complex.

\section{Introduction}

Hyperspectral imaging [1] is concerned with the measurement, analysis, and interpretation of spectra acquired from a given scene (or specific object) at a short, medium, or long distance by an airborne or satellite sensor [2]. Hyperspectral imaging instruments such as the NASA Jet Propulsion Laboratory's Airborne Visible Infrared Imaging Spectrometer (AVIRIS) [3] are now able to record the visible and near-infrared spectrum (wavelength region from 0.4 to 2.5 micrometers) of the reflected light of an area 2 to 12 kilometers wide and several kilometers long using 224 spectral bands. The resulting "image cube" (see Figure 1) is a stack of images in which each pixel (vector) has an associated spectral signature or fingerprint that uniquely characterizes the underlying objects [4]. The resulting data volume typically comprises several GBs per flight [5].

The special properties of hyperspectral data have significantly expanded the domain of many analysis techniques, including (supervised and unsupervised) classification, spectral unmixing, compression, target, and anomaly detection
[6-10]. Specifically, the automatic detection of targets and anomalies is highly relevant in many application domains, including those addressed in Figure 2 [11-13]. For instance, automatic target and anomaly detection are considered very important tasks for hyperspectral data exploitation in defense and security applications [14, 15]. During the last few years, several algorithms have been developed for the aforementioned purposes, including the automatic target detection and classification (ATDCA) algorithm [12], an unsupervised fully constrained least squares (UFCLSs) algorithm [16], an iterative error analysis (IEA) algorithm [17], or the well-known RX algorithm developed by Reed and $\mathrm{Yu}$ for anomaly detection [18]. The ATDCA algorithm finds a set of spectrally distinct target pixels vectors using the concept of orthogonal subspace projection (OSP) [19] in the spectral domain. On the other hand, the UFCLS algorithm generates a set of distinct targets using the concept of least square-based error minimization. The IEA uses a similar approach, but with a different initialization condition. The RX algorithm is based on the application of a so-called RXD filter, given by the well-known Mahalanobis distance. Many 


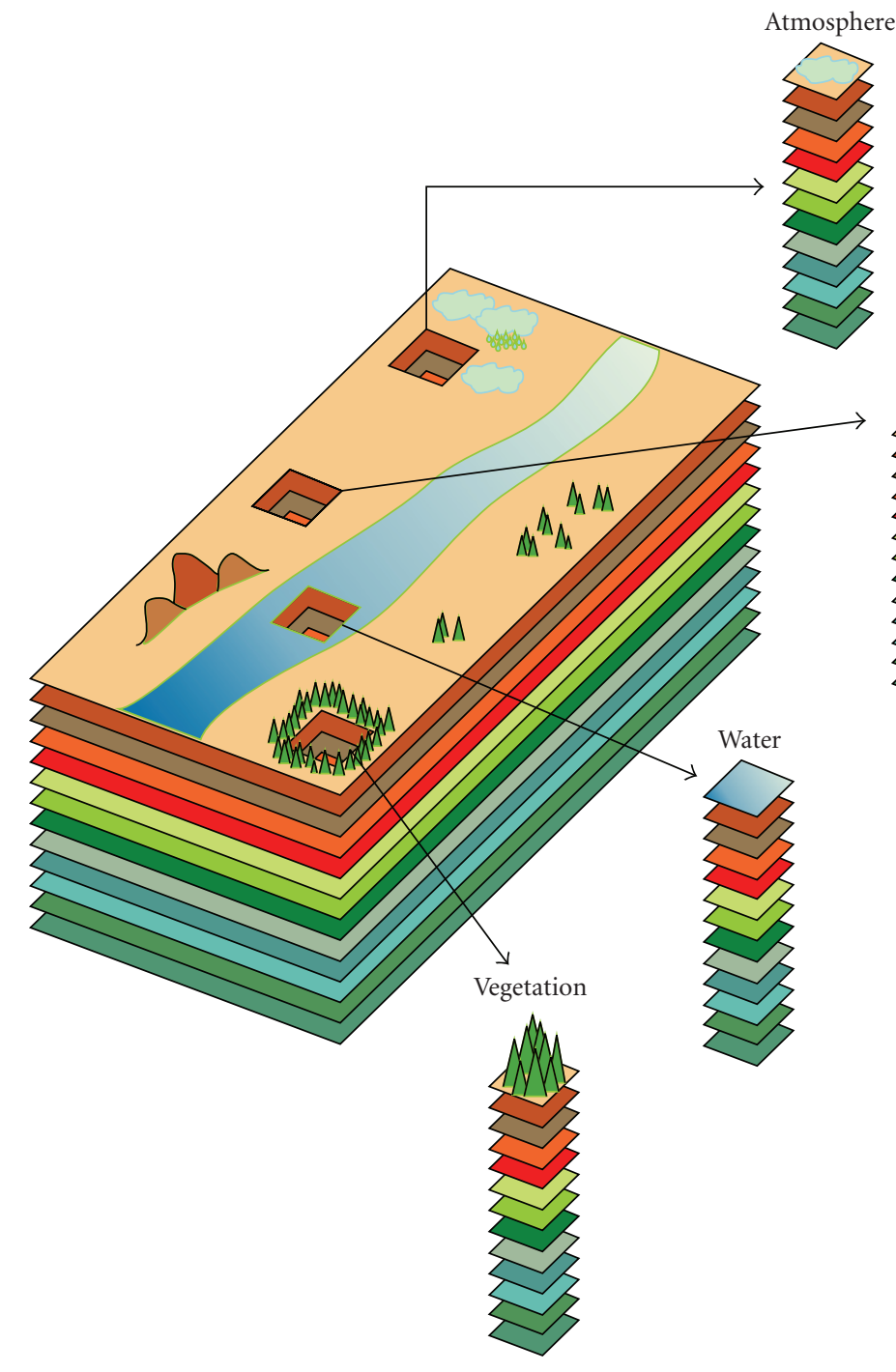

(a)
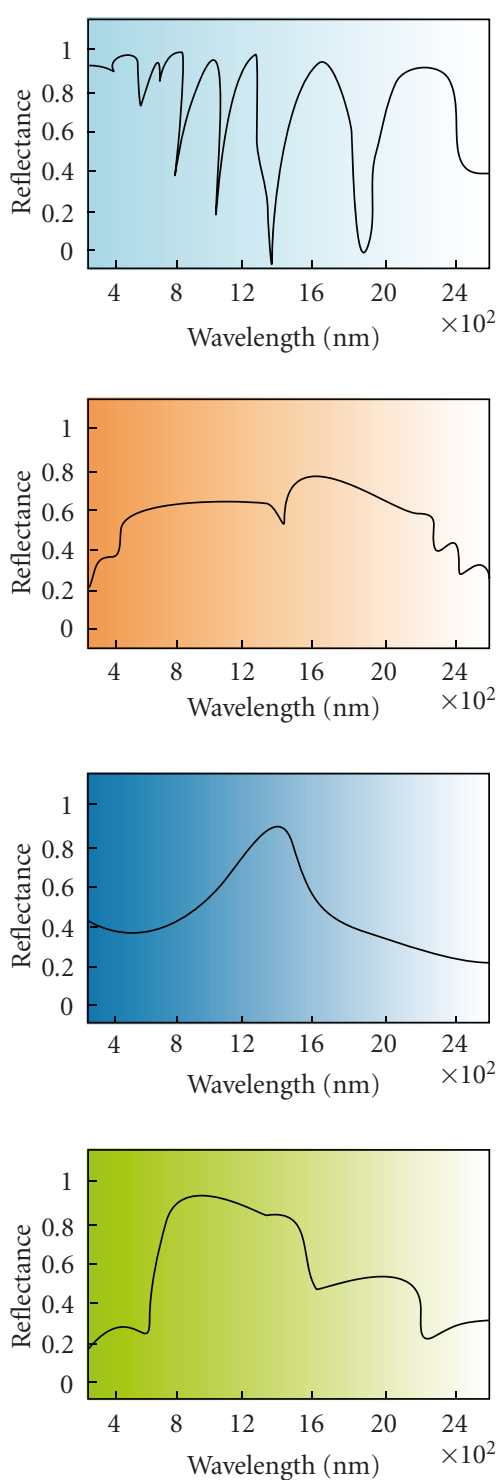

(b)

FIGURE 1: Concept of hyperspectral imaging.

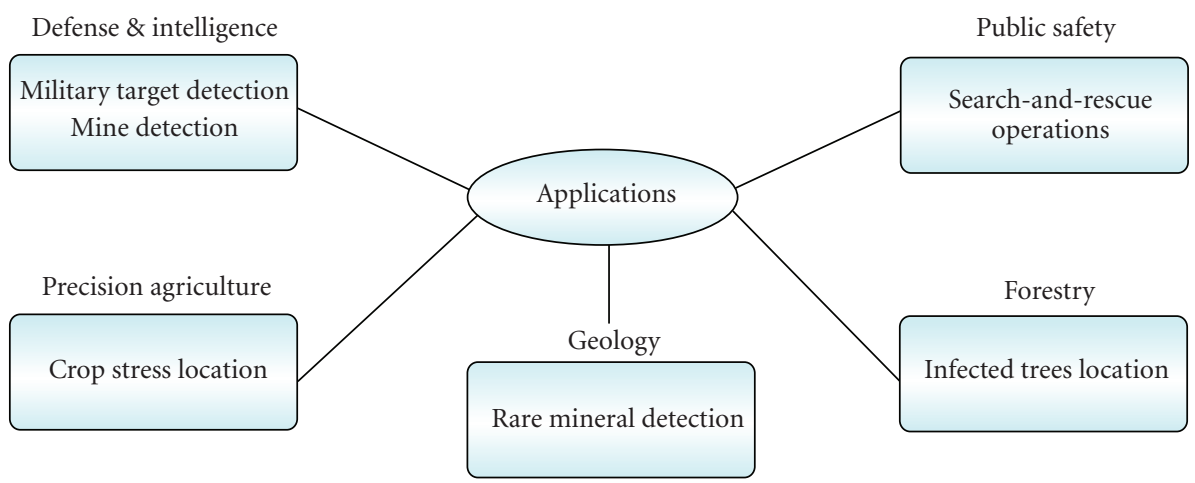

FIGURE 2: Applications of target and anomaly detection. 
other target/anomaly detection algorithms have also been proposed in the recent literature, using different concepts such as background modeling and characterization $[13,20]$.

Depending on the complexity and dimensionality of the input scene [21], the aforementioned algorithms may be computationally very expensive, a fact that limits the possibility of utilizing those algorithms in time-critical applications [5]. In turn, the wealth of spectral information available in hyperspectral imaging data opens groundbreaking perspectives in many applications, including target detection for military and defense/security deployment [22]. In particular, algorithms for detecting (moving or static) targets or targets that could expand their size (such as propagating fires) often require timely responses for swift decisions that depend upon high computing performance of algorithm analysis [23]. Therefore, in many applications it is of critical importance that automatic target and anomaly detection algorithms complete their analysis tasks quickly enough for practical use. Despite the growing interest in parallel hyperspectral imaging research [24-26], only a few parallel implementations of automatic target and anomaly detection algorithms for hyperspectral data exist in the open literature [14]. However, with the recent explosion in the amount and dimensionality of hyperspectral imagery, parallel processing is expected to become a requirement in most remote sensing missions [5], including those related with the detection of anomalous and/or concealed targets. Of particular importance is the design of parallel algorithms able to detect target and anomalies at subpixel levels [22], thus overcoming the limitations imposed by the spatial resolution of the imaging instrument.

In the past, Beowulf-type clusters of computers have offered an attractive solution for fast information extraction from hyperspectral data sets already transmitted to Earth [27-29]. The goal was to create parallel computing systems from commodity components to satisfy specific requirements for the Earth and space sciences community. However, these systems are generally expensive and difficult to adapt to on-board data processing scenarios, in which low-weight and low-power integrated components are essential to reduce mission payload and obtain analysis results in real-time, that is, at the same time as the data is collected by the sensor. In this regard, an exciting new development in the field of commodity computing is the emergence of commodity graphic processing units (GPUs), which can now bridge the gap towards on-board processing of remotely sensed hyperspectral data $[15,30]$. The speed of graphics hardware doubles approximately every six months, which is much faster than the improving rate of the CPUs (even those made up by multiple cores) which are interconnected in a cluster. Currently, state-of-the-art GPUs deliver peak performances more than one order of magnitude over high-end microprocessors. The ever-growing computational requirements introduced by hyperspectral imaging applications can fully benefit from this type of specialized hardware and take advantage of the compact size and relatively low cost of these units, which make them appealing for on-board data processing at lower costs than those introduced by other hardware devices [5].
In this paper, we develop and compare several new computationally efficient parallel versions (for clusters and GPUs) of two highly representative algorithms for target (ATDCA) and anomaly detection (RX) in hyperspectral scenes. In the case of ATDCA, we use several distance metrics in addition to the OSP approach implemented in the original algorithm. The considered metrics include the spectral angle distance (SAD) and the spectral information divergence (SID), which introduce an innovation with regards to the distance criterion for target selection originally available in the ATDCA algorithm. The parallel versions are quantitatively and comparatively analyzed (in terms of target detection accuracy and parallel performance) in the framework of a real defense and security application, focused on identifying thermal hot spots (which can be seen as targets and/or anomalies) in a complex urban background, using AVIRIS hyperspectral data collected over the World Trade Center in New York just five days after the terrorist attack of September 11th, 2001.

The remainder of the paper is organized as follows. Section 2 describes the considered target (ATDCA) and anomaly (RX) detection algorithms. Section 3 develops parallel implementations (referred to as P-ATDCA and P-RX, resp.) for clusters of computers. Section 4 develops parallel implementations (referred to as G-ATDCA and G-RX, resp.) for GPUs. Section 5 describes the hyperspectral data set used for experiments and then discusses the experimental results obtained in terms of both target/anomaly detection accuracy and parallel performance, using a Beowulf cluster with 256 processors available at NASA's Goddard Space Flight Center in Maryland and a NVidia GeForce 9800 GX2 GPU. Finally, Section 6 concludes with some remarks and hints at plausible future research.

\section{Methods}

In this section we briefly describe the target detection algorithms that will be efficiently implemented in parallel (using different high-performance computing architectures) in this work. These algorithms are the ATDCA for automatic target and classification and the RX for anomaly detection. In the former case, several distance measures are described for implementation of the algorithm.

2.1. ATDCA Algorithm. The ATDCA algorithm [12] was developed to find potential target pixels that can be used to generate a signature matrix used in an orthogonal subspace projection (OSP) approach [19]. Let $\mathbf{x}_{0}$ be an initial target signature (i.e., the pixel vector with maximum length). The ATDCA begins by an orthogonal subspace projector specified by the following expression:

$$
P_{\mathbf{U}}^{\perp}=\mathbf{I}-\mathbf{U}\left(\mathbf{U}^{T} \mathbf{U}\right)^{-1} \mathbf{U}^{T},
$$

which is applied to all image pixels, with $\mathbf{U}=\left[\mathbf{x}_{0}\right]$. It then finds a target signature, denoted by $\mathbf{x}_{1}$, with the maximum projection in $\left\langle\mathbf{x}_{0}\right\rangle^{\perp}$, which is the orthogonal complement space linearly spanned by $\mathbf{x}_{0}$. A second target signature $\mathbf{x}_{2}$ can then be found by applying another orthogonal subspace 
projector $P_{\mathbf{U}}^{\perp}$ with $\mathbf{U}=\left[\mathbf{x}_{0}, \mathbf{x}_{1}\right]$ to the original image, where the target signature that has the maximum orthogonal projection in $\left\langle\mathbf{x}_{0}, \mathbf{x}_{1}\right\rangle^{\perp}$ is selected as $\mathbf{x}_{2}$. The above procedure is repeated until a set of target pixels $\left\{\mathbf{x}_{0}, \mathbf{x}_{1}, \ldots, \mathbf{x}_{t}\right\}$ is extracted, where $t$ is an input parameter to the algorithm.

In addition to the standard OSP approach, we have explored other alternatives in the implementation of ATDCA, given by replacing the $P_{\mathrm{U}}^{\perp}$ operator used in the OSP implementation by one of the distance measures described as follows [31, 32]:

(i) the 1-Norm between two pixel vectors $\mathbf{x}_{i}$ and $\mathbf{x}_{j}$, defined by $\left\|\mathbf{x}_{i}-\mathbf{x}_{j}\right\|$,

(ii) the 2-Norm between two pixel vectors $\mathbf{x}_{i}$ and $\mathbf{x}_{j}$, defined by $\left\|\mathbf{x}_{i}-\mathbf{x}_{j}\right\|_{2}$,

(iii) the Infinity-Norm between two pixel vectors $\mathbf{x}_{i}$ and $\mathbf{x}_{j}$, defined by $\left\|\mathbf{x}_{i}-\mathbf{x}_{j}\right\|_{\infty}$,

(iv) the spectral angle distance (SAD) between two pixel vectors $\mathbf{x}_{i}$ and $\mathbf{x}_{j}$, defined by the following expression [4]: $\operatorname{SAD}\left(\mathbf{x}_{i}, \mathbf{x}_{j}\right)=\cos ^{-1}\left(\mathbf{x}_{i} \cdot \mathbf{x}_{j} /\left\|\mathbf{x}_{i}\right\|_{2} \cdot\left\|\mathbf{x}_{j}\right\|_{2}\right)$; as opposed to the previous metric, $\mathrm{SAD}$ is invariant in the presence of illumination interferers, which can provide advantages in terms of target and anomaly detection in complex backgrounds,

(v) the spectral information divergence (SID) between two pixel vectors $\mathbf{x}_{i}$ and $\mathbf{x}_{j}$, defined by the following expression [4]: $\operatorname{SID}\left(\mathbf{x}_{i}, \mathbf{x}_{j}\right)=D\left(\mathbf{x}_{i} \| \mathbf{x}_{j}\right)+D\left(\mathbf{x}_{j} \| \mathbf{x}_{i}\right)$, where $D\left(\mathbf{x}_{i} \| \mathbf{x}_{j}\right)=\sum_{k=1}^{n} p_{k} \cdot \log \left(p_{k} / q_{k}\right)$. Here, we define $p_{k}=x_{i}^{(k)} / \sum_{k=1}^{n} x_{i}^{(k)}$ and $q_{k}=x_{j}^{(k)} / \sum_{k=1}^{n} x_{j}^{(k)}$.

2.2. $R X$ Algorithm. The $\mathrm{RX}$ algorithm has been widely used in signal and image processing [18]. The filter implemented by this algorithm is referred to as RX filter (RXF) and defined by the following expression:

$$
\delta^{\mathrm{RXF}}(\mathbf{x})=(\mathbf{x}-\mu)^{T} \mathbf{K}^{-1}(\mathbf{x}-\mu),
$$

where $\mathbf{x}=\left[x^{(0)}, x^{(1)}, \ldots, x^{(n)}\right]$ is a sample, $n$-dimensional hyperspectral pixel (vector), $\mu$ is the sample mean, and $\mathbf{K}$ is the sample data covariance matrix. As we can see, the form of $\delta^{\mathrm{RXF}}$ is actually the well-known Mahalanobis distance [8]. It is important to note that the images generated by the RX algorithm are generally gray-scale images. In this case, the anomalies can be categorized in terms of the value returned by RXF, so that the pixel with higher value of $\delta^{\mathrm{RXF}}(\mathbf{x})$ can be considered the first anomaly, and so on.

\section{Parallel Implementations for Clusters of Computers}

Clusters of computers are made up of different processing units interconnected via a communication network [33]. In previous work, it has been reported that data-parallel approaches, in which the hyperspectral data is partitioned among different processing units, are particularly effective for parallel processing in this type of high-performance computing systems $[5,26,28]$. In this framework, it is

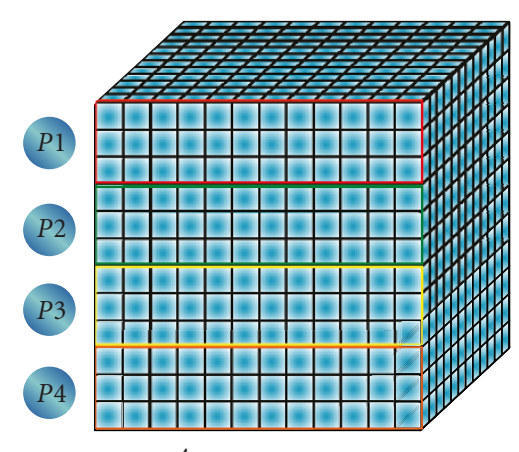

4 processors

(a)

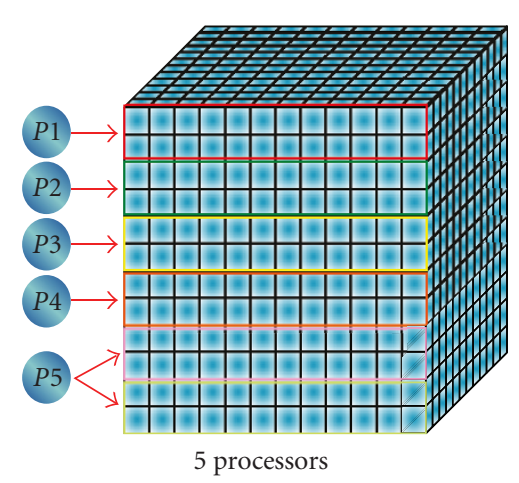

(b)

FIGURE 3: Spatial-domain decomposition of a hyperspectral data set into four (a) and five (b) partitions.

very important to define the strategy for partitioning the hyperspectral data. In our implementations, a data-driven partitioning strategy has been adopted as a baseline for algorithm parallelization. Specifically, two approaches for data partitioning have been tested [28].

(i) Spectral-domain partitioning. This approach subdivides the multichannel remotely sensed image into small cells or subvolumes made up of contiguous spectral wavelengths for parallel processing.

(ii) Spatial-domain partitioning. This approach breaks the multichannel image into slices made up of one or several contiguous spectral bands for parallel processing. In this case, the same pixel vector is always entirely assigned to a single processor, and slabs of spatially adjacent pixel vectors are distributed among the processing nodes (CPUs) of the parallel system. Figure 3 shows two examples of spatialdomain partitioning over 4 processors and over 5 processors, respectively.

Previous experimentation with the above-mentioned strategies indicated that spatial-domain partitioning can significantly reduce inter-processor communication, resulting from the fact that a single pixel vector is never partitioned and communications are not needed at the pixel level [28]. In the following, we assume that spatial-domain decomposition is always used when partitioning the hyperspectral data 
cube. The inputs to the considered parallel algorithms are a hyperspectral image cube $\mathbf{F}$ with $n$ dimensions, where $\mathbf{x}$ denotes the pixel vector of the same scene, and a maximum number of targets to be detected, $t$. The output in all cases is a set of target pixel vectors $\left\{\mathbf{x}_{1}, \mathbf{x}_{2}, \ldots, \mathbf{x}_{t}\right\}$.

3.1. P-ATDCA. The parallel version of ATDCA adopts the spatial-domain decomposition strategy depicted in Figure 3 for dividing the hyperspectral data cube in master-slave fashion. The algorithm has been implemented in the $\mathrm{C}++$ programming language using calls to MPI, the message passing interface library commonly available for parallel implementations in multiprocessor systems (http://www.mcs.anl.gov/research/projects/mpi). The parallel implementation, denoted by P-ATDCA and summarized by a diagram in Figure 4, consists of the following steps.

(1) The master divides the original image cube $\mathbf{F}$ into $P$ spatial-domain partitions. Then, the master sends the partitions to the workers.

(2) Each worker finds the brightest pixel in its local partition (local maximum) using $\mathbf{x}_{1}=\arg \max \left\{\mathbf{x}^{T}\right.$. $\mathbf{x}$, where the superscript $T$ denotes the vector transpose operation. Each worker then sends the spatial locations of the pixel identified as the brightest one in its local partition back to the master. For illustrative purposes, Figure 5 shows the piece of $\mathrm{C}++$ code that the workers execute in order to send their local maxima to the master node using the MPI function MPI_send. Here, localmax is the local maximum at the node given by identifier node_id, where node $\_i d=0$ for the master and node $i d>0$ for the workers. MPI_COMM_WORLD is the name of the communicator or collection of processes that are running concurrently in the system (in our case, all the different parallel tasks allocated to the $P$ workers).

(3) Once all the workers have completed their parts and sent their local maxima, the master finds the brightest pixel of the input scene (global maximum), $\mathbf{x}_{1}$, by applying the arg max operator in step 2 to all the pixels at the spatial locations provided by the workers, and selecting the one that results in the maximum score. Then, the master sets $\mathbf{U}=\mathbf{x}_{1}$ and broadcasts this matrix to all workers. As shown by Figure 5, this is implemented (in the workers) by a call to MPI_Recv that stops the worker until the value of the global maximum globalmax is received from the master. On the other hand, Figure 6 shows the code designed for calculation of the global maximum at the master. First, the master receives all the local maxima from the workers using the MPI_Gather function. Then, the worker which contains the global maximum out of the local maxima is identified in the for loop. Finally, the global maximum is broadcast to all the workers using the MPI_Bcast function.

(4) After this process is completed, each worker now finds (in parallel) the pixel in its local partition with the maximum orthogonal projection relative to the pixel vectors in $\mathbf{U}$, using a projector given by $P_{\mathbf{U}}^{\perp}=$ $\mathbf{I}-\mathbf{U}\left(\mathbf{U}^{T} \mathbf{U}\right)^{-1} \mathbf{U}^{T}$, where $\mathbf{U}$ is the identity matrix. The orthogonal space projector $P_{\mathbf{U}}^{\perp}$ is now applied to all pixel vectors in each local partition to identify the most distinct pixels (in orthogonal sense) with regards to the previously detected ones. Each worker then sends the spatial location of the resulting local pixels to the master node.

(5) The master now finds a second target pixel by applying the $P_{\mathbf{U}}^{\perp}$ operator to the pixel vectors at the spatial locations provided by the workers, and selecting the one which results in the maximum score as follows: $\mathbf{x}_{2}=\arg \max \left\{\left(P_{\mathbf{U}}^{\perp} \mathbf{x}\right)^{T}\left(P_{\mathbf{U}}^{\perp} \mathbf{x}\right)\right\}$. The master sets $\mathbf{U}=\left\{\mathbf{x}_{1}, \mathbf{x}_{2}\right\}$ and broadcasts this matrix to all workers.

(6) Repeat from step 4 until a set of $t$ target pixels, $\left\{\mathbf{x}_{1}, \mathbf{x}_{2}, \ldots, \mathbf{x}_{t}\right\}$, are extracted from the input data. It should be noted that the P-ATDCA algorithm has not only been implemented using the aforementioned OSP-based approach, but also the different metrics discussed in Section 2.2 by simply replacing the $P_{\mathrm{U}}^{\perp}$ operator by a different distance measure.

3.2. P-RX. Our MPI-based parallel version of the RX algorithm for anomaly detection also adopts the spatialdomain decomposition strategy depicted in Figure 3. The parallel algorithm is given by the following steps, which are graphically illustrated in Figure 7.

(1) The master processor divides the original image cube F into $P$ spatial-domain partitions and distributes them among the workers.

(2) The master calculates the $n$-dimensional mean vector $\mathbf{m}$ concurrently, where each component is the average of the pixel values of each spectral band of the unique set. This vector is formed once all the processors finish their parts. At the same time, the master also calculates the sample spectral covariance matrix $\mathbf{K}$ concurrently as the average of all the individual matrices produced by the workers using their respective portions. This procedure is described in detail in Figure 7.

(3) Using the above information, each worker applies (locally) the RXF filter given by the Mahalanobis distance to all the pixel vectors in the local partition as follows: $\delta^{(\mathrm{RXF})}(\mathbf{x})=(\mathbf{x}-\mathbf{m})^{T} \mathbf{K}^{-1}(\mathbf{x}-\mathbf{m})$ and returns the local result to the master. At this point, it is very important to emphasize that, once the sample covariance matrix is calculated in parallel as indicated by Figure 7, the inverse needed for the local computations at the workers is calculated serially at each node.

(4) The master now selects the $t$ pixel vectors with higher associated value of $\delta^{(\mathrm{RXF})}$ and uses them to form a final set of targets $\left\{\mathbf{x}_{1}, \mathbf{x}_{2}, \ldots, \mathbf{x}_{t}\right\}$. 


\section{Parallel Implementations for GPUs}

GPUs can be abstracted in terms of a stream model, under which all data sets are represented as streams (i.e., ordered data sets) [30]. Algorithms are constructed by chaining socalled kernels, which operate on entire streams, taking one or more streams as inputs and producing one or more streams as outputs. Thereby, data-level parallelism is exposed to hardware, and kernels can be concurrently applied. Modern GPU architectures adopt this model and implement a generalization of the traditional rendering pipeline, which consists of two main stages [5].

(1) Vertexprocessing. The input to this stage is a stream of vertices from a $3 \mathrm{D}$ polygonal mesh. Vertex processors transform the $3 \mathrm{D}$ coordinates of each vertex of the mesh into a 2D screen position and apply lighting to determine their colors (this stage is fully programmable).

(2) Fragment processing. In this stage, the transformed vertices are first grouped into rendering primitives, such as triangles, and scan-converted into a stream of pixel fragments. These fragments are discrete portions of the triangle surface that corresponds to the pixels of the rendered image. Apart from identifying constituent fragments, this stage also interpolates attributes stored at the vertices, such as texture coordinates, and stores the interpolated values at each fragment. Arithmetical operations and texture lookups are then performed by fragment processors to determine the ultimate color for the fragment. For this purpose, texture memories can be indexed with different texture coordinates, and texture values can be retrieved from multiple textures.

It should be noted that fragment processors currently support instructions that operate on vectors of four RGBA components (Red/Green/Blue/Alpha channels) and include dedicated texture units that operate with a deeply pipelined texture cache. As a result, an essential requirement for mapping nongraphics algorithms onto GPUs is that the data structure can be arranged according to a streamflow model, in which kernels are expressed as fragment programs and data streams are expressed as textures. Using C-like, high-level languages such as NVidia compute unified device architecture (CUDA), programmers can write fragment programs to implement general-purpose operations. CUDA is a collection of $\mathrm{C}$ extensions and a runtime library (http:// www.nvidia.com/object/cuda_home.html). CUDA's functionality primarily allows a developer to write $\mathrm{C}$ functions to be executed on the GPU. CUDA also includes memory management and execution configuration, so that a developer can control the number of GPU processors and processing threads that are to be invoked during a function's execution.

The first issue that needs to be addressed is how to map a hyperspectral image onto the memory of the GPU. Since the size of hyperspectral images usually exceeds the capacity of such memory, we split them into multiple spatial-domain partitions [28] made up of entire pixel vectors (see Figure 3);

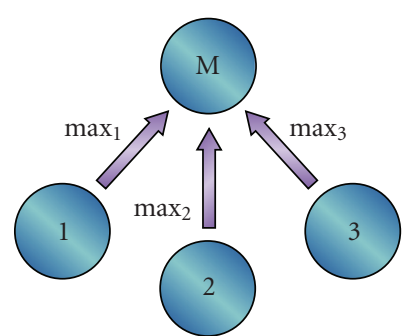
pixel in its local partition and sends it to the master

(a)

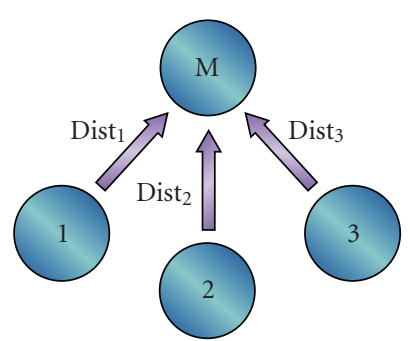

3) Workers find local pixel with maximum distance with regards to previous pixels

(c)
1) Workers find the brightest

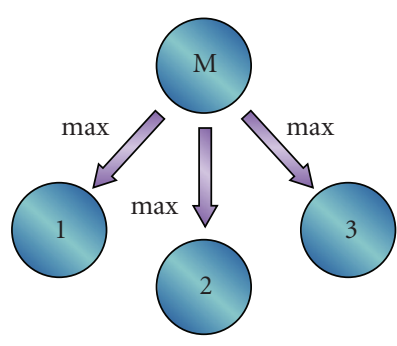

2) Master broadcast the brightest pixel to all workers

(b)

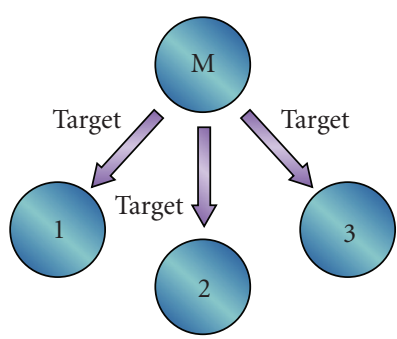

4) Repeat the process until a set of $t$ targets have been identified after subsequent iterations

(d)
Figure 4: Graphical summary of the parallel implementation of ATDCA algorithm using 1 master processor and 3 slaves.

that is, as in our cluster-based implementations, each spatialdomain partition incorporates all the spectral information on a localized spatial region and is composed of spatially adjacent pixel vectors. Each spatial-domain partition is further divided into 4-band tiles (called spatial-domain tiles), which are arranged in different areas of a 2D texture [30]. Such partitioning allows us to map four consecutive spectral bands onto the RGBA color channels of a texture element. Once the procedure adopted for data partitioning has been described, we provide additional details about the GPU implementations of RX and ATDCA algorithms, referred to hereinafter as G-RX and G-ATDCA, respectively.

4.1. G-ATDCA. Our GPU version of the ATDCA algorithm for target detection is given by the following steps.

(1) Once the hyperspectral image is mapped onto the GPU memory, a structure (grid) in which the number of blocks equals the number of lines in the hyperspectral image and the number of threads equals the number of samples is created, thus making sure that all pixels in the hyperspectral image are processed in parallel (if this is not possible due to limited memory resources in the GPU, CUDA automatically performs several iterations, each of which processes as many pixels as possible in parallel).

(2) Using the aforementioned structure, calculate the brightest pixel $\mathbf{x}_{1}$ in the original hyperspectral scene by means of a CUDA kernel which performs part of 
the calculations to compute $\mathbf{x}_{1}=\arg \max \left\{\mathbf{x}^{T} \cdot \mathbf{x}\right\}$ after computing (in parallel) the dot product between each pixel vector $\mathbf{x}$ in the original hyperspectral image and its own transposed version $\mathbf{x}^{T}$. For illustrative purposes, Figure 8 shows a portion of code which includes the definition of the number of blocks numBlocks and the number of processing threads per block numThreadsPerBlock, and then calls the CUDA kernel BrightestPixel that computes the value of $\mathbf{x}_{1}$. Here, d_bright_matrix is the structure that stores the output of the computation $\mathbf{x}^{T} \cdot \mathbf{x}$ for each pixel. Figure 9 shows the code of the CUDA kernel BrightestPixel, in which each different thread computes a different value of $\mathbf{x}^{T} \cdot \mathbf{x}$ for a different pixel (each thread is given by an identification number $i d x$, and there are as many concurrent threads as pixels in the original hyperspectral image). Once all the concurrent threads complete their calculations, the G-ATDCA implementation simply computes the value in d_bright_matrix with maximum associated value and obtains the pixel in that position, labeling the pixel as $\mathbf{x}_{1}$. Although this operation is inevitably sequential, it is performed in the GPU.

(3) Once the brightest pixel in the original hyperspectral image has been identified as the first target $\mathbf{U}=\mathbf{x}_{1}$, the ATDCA algorithm is executed in the GPU by means of another kernel in which the number of blocks equals the number of lines in the hyperspectral image and the number of threads equals the number of samples is created, thus making sure that all pixels in the hyperspectral image are processed in parallel. The concurrent threads find (in parallel) the values obtained after applying the OSP-based projection operator $P_{\mathbf{U}}^{\perp}=\mathbf{I}-\mathbf{U}\left(\mathbf{U}^{T} \mathbf{U}\right)^{-1} \mathbf{U}^{T}$ to each pixel (using the structure d_bright_matrix to store the resulting projection values), and then the GATDCA algorithm finds a second target pixel from the values stored in d_bright matrix as follows: $\mathbf{x}_{2}=\arg \max \left\{\left(P_{\mathbf{U}}^{\perp} \mathbf{x}\right)^{T}\left(P_{\mathbf{U}}^{\perp} \mathbf{x}\right)\right\}$. The procedure is repeated until a set of $t$ target pixels, $\left\{\mathbf{x}_{1}, \mathbf{x}_{2}, \ldots, \mathbf{x}_{t}\right\}$, are extracted from the input data. Although in this description we have only referred to the OSPbased operation, the different metrics discussed in Section 2.2 have been implemented by devising different kernels which can be replaced in our GATDCA implementation in plug and play fashion in order to modify the distance measure used by the algorithm to identify new targets along the process.

4.2. G-RX. Our GPU version of the RX algorithm for anomaly detection is given by the following steps.

(1) Once the hyperspectral image is mapped onto the GPU memory, a structure (grid) containing $n$ blocks of threads, each containing $n$ processing threads, is defined using CUDA. As a result, a total of $n \times n$ processing threads are available.
(2) Using the aforementioned structure, calculate the sample spectral covariance matrix $\mathbf{K}$ in parallel by means of a CUDA kernel which performs the calculations needed to compute $\delta^{(\mathrm{RXF})}(\mathbf{x})=(\mathbf{x}-$ $\mathbf{m})^{T} \mathbf{K}^{-1}(\mathbf{x}-\mathbf{m})$ for each pixel $\mathbf{x}$. For illustrative purposes, Figure 10 shows a portion of code which includes the initialization of matrix $\mathbf{K}$ in the GPU memory using cudaMemset, a call to the CUDA kernel RXGPU designed to calculate $\delta^{(\mathrm{RXF})}$, and finally a call to cudaThreadSynchronize to make sure that the initiated threads are synchronized. Here, d_hyper_image is the original hyperspectral image, $d_{-} K$ denotes the matrix $\mathbf{K}$, and numlines, numsamples, and numbands, respectively denote the number of lines, samples, and bands of the original hyperspectral image. It should be noted that the RXGPU kernel implements the Gauss-Jordan elimination method for calculating $\mathbf{K}^{-1}$. We recall that the entire image data is allocated in the GPU memory, and therefore it is not necessary to partition the data as it was the case in the cluster-based implementation. In fact, this is one of the main advantages of GPUs over clusters of computers (GPUs are shared memory architectures, while clusters are generally distributed memory architectures in which message passing is needed to distribute the workload among the workers). A particularity of the Gauss-Jordan elimination method is that it converts the source matrix into an identity matrix pivoting, where the pivot is the element in the diagonal of the matrix by which other elements are divided in an algorithm. The GPU naturally parallelizes the pivoting operation by applying the calculation at the same time to many rows and columns, and hence the inverse operation is calculated in parallel in the GPU.

(3) Once the $\delta^{(\mathrm{RXF})}$ has been computed (in parallel) for every pixel $\mathbf{x}$ in the original hyperspectral image, a final (also parallel) step selects the $t$ pixel vectors with higher associated value of $\delta^{(\mathrm{RXF})}$ (stored in d_result) and uses them to form a final set of targets $\left\{\mathbf{x}_{1}, \mathbf{x}_{2}, \ldots, \mathbf{x}_{t}\right\}$. This is done using the portion of code illustrated in Figure 11, which calls a CUDA kernel RXResult which implements this functionality. Here, the number of blocks numBlocks equals the number of lines in the hyperspectral image, while the number of threads numThreadsPerBlock equals the number of samples, thus making sure that all pixels in the hyperspectral image are processed in parallel (if this is not possible due to limited memory resources in the GPU, CUDA automatically performs several iterations, each of which processes as many pixels as possible in parallel).

\section{Experimental Results}

This section is organized as follows. In Section 5.1 we describe the AVIRIS hyperspectral data set used in our experiments. Section 5.2 describes the parallel computing 


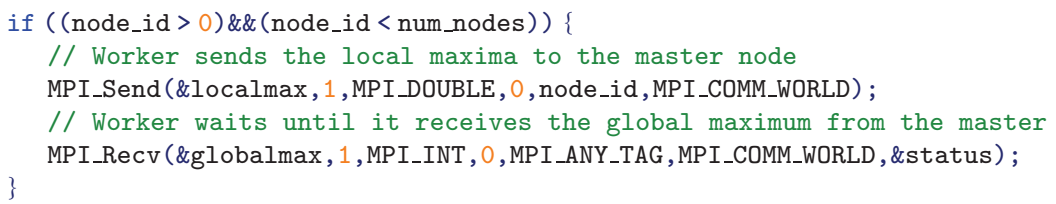

FIGURE 5: Portion of the code of a worker in our P-ATDCA implementation, in which the worker sends a precomputed local maximum to the master and waits for a global maximum from the master.

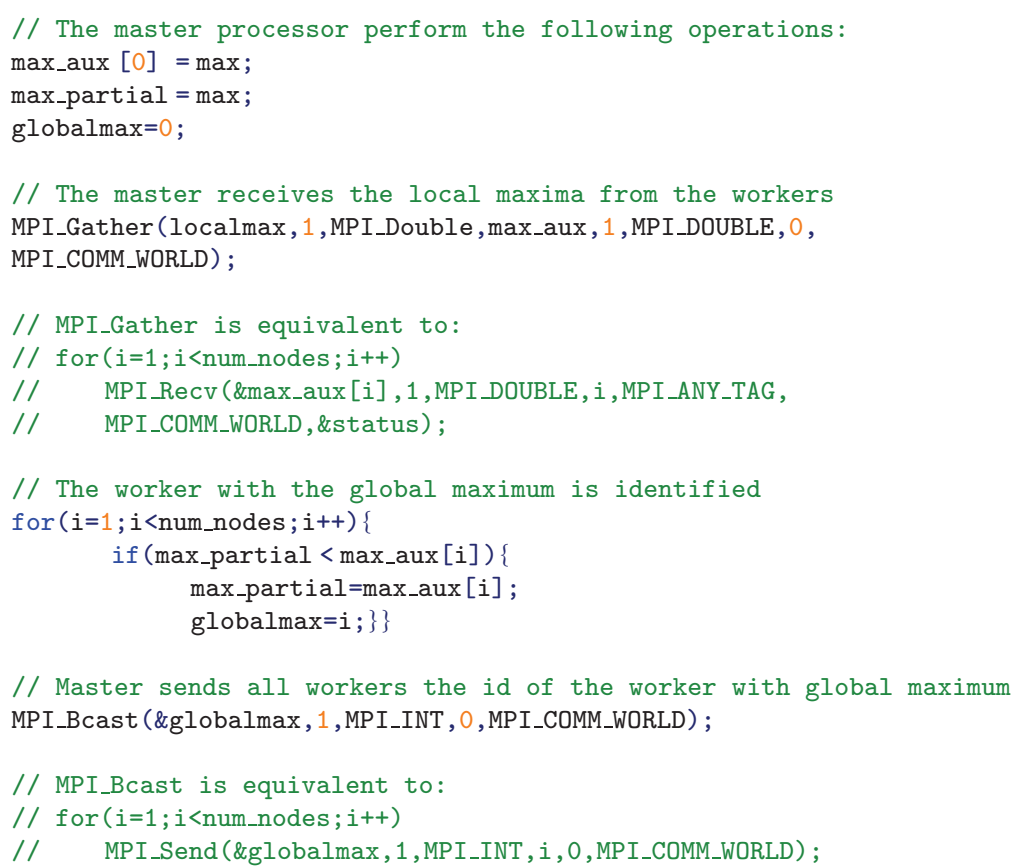

Figure 6: Portion of the code of the master in our P-ATDCA implementation, in which the master receives the local maxima from the workers, computes a global maximum, and sends all workers the id of the worker which contains the global maximum.

platforms used for experimental evaluation, which comprise a Beowulf cluster at NASA's Goddard Space Flight Center in Maryland and an NVidia GeForce 8900 GX2 GPU. Section 5.3 discusses the target and anomaly detection accuracy of the parallel algorithms when analyzing the hyperspectral data set described in Section 5.1. Section 5.4 describes the parallel performance results obtained after implementing the P-ATDCA and P-RX algorithms on the Beowulf cluster. Section 5.5 describes the parallel performance results obtained after implementing the G-ATDCA and G-RX algorithms on the GPU. Finally, Section 5.6 provides a comparative assessment and general discussion of the different parallel algorithms presented in this work in light of the specific characteristics of the considered parallel platforms (clusters versus GPUs).

5.1. Data Description. The image scene used for experiments in this work was collected by the AVIRIS instrument, which was flown by NASA's Jet Propulsion Laboratory over the World Trade Center (WTC) area in New York City on September 16, 2001, just five days after the terrorist attacks that collapsed the two main towers and other buildings in the WTC complex. The full data set selected for experiments consists of $614 \times 512$ pixels, 224 spectral bands, and a total size of (approximately) $140 \mathrm{MB}$. The spatial resolution is 1.7 meters per pixel. The leftmost part of Figure 12 shows a false color composite of the data set selected for experiments using the 1682, 1107, and $655 \mathrm{~nm}$ channels, displayed as red, green, and blue, respectively. Vegetated areas appear green in the leftmost part of Figure 12, while burned areas appear dark gray. Smoke coming from the WTC area (in the red rectangle) and going down to south Manhattan appears bright blue due to high spectral reflectance in the $655 \mathrm{~nm}$ channel.

Extensive reference information, collected by U.S. Geological Survey (USGS), is available for the WTC scene (http://speclab.cr.usgs.gov/wtc). In this work, we use 


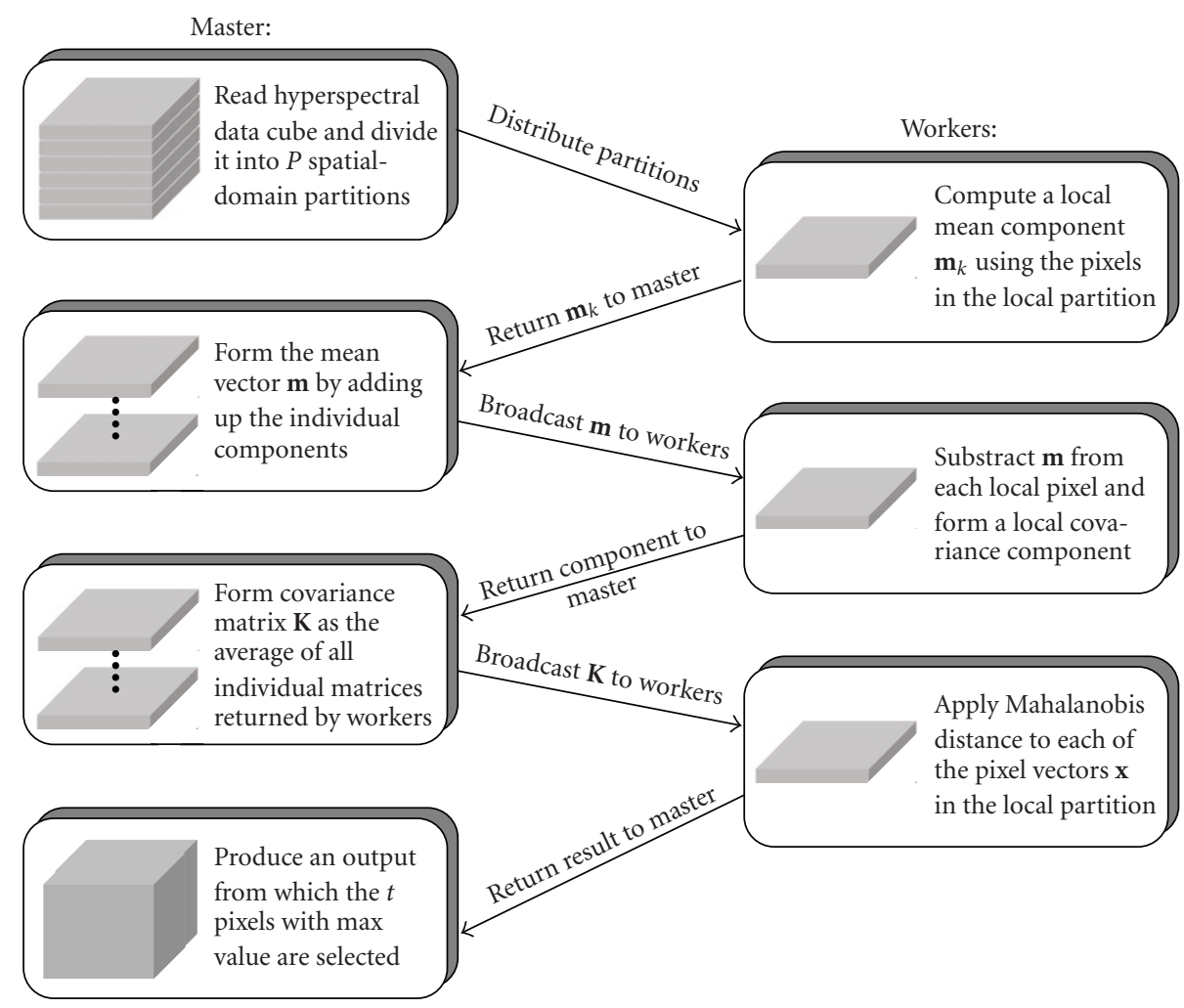

FIGURE 7: Parallel implementation of the RX algorithm in clusters of computers.

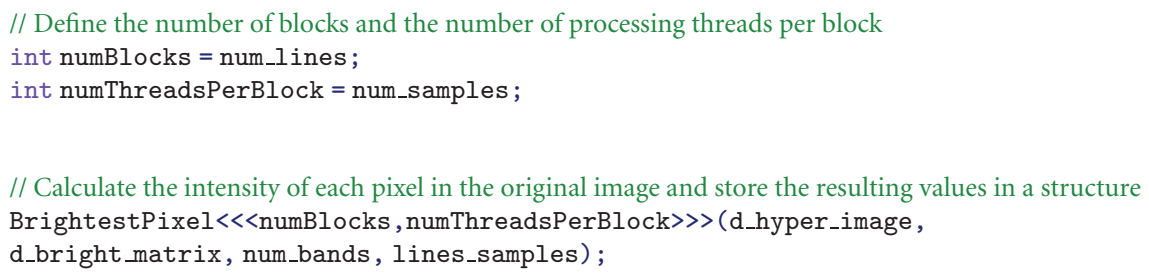

FIgURE 8: Portion of code which calls the CUDA kernel BrightestPixel that computes (in parallel) the brightest pixel in the scene in the G-ATDCA implementation.

a U.S. Geological Survey thermal map (http://pubs.usgs .gov/of/2001/ofr-01-0429/hotspot.key.tgif.gif) which shows the target locations of the thermal hot spots at the WTC area, displayed as bright red, orange, and yellow spots at the rightmost part of Figure 12. The map is centered at the region where the towers collapsed, and the temperatures of the targets range from $700 \mathrm{~F}$ to $1300 \mathrm{~F}$. Further information available from USGS about the targets (including location, estimated size, and temperature) is reported on Table 1. As shown by Table 1, all the targets are subpixel in size since the spatial resolution of a single pixel is 1.7 square meters. The thermal map displayed in the rightmost part of Figure 12 will be used in this work as ground-truth to validate the target detection accuracy of the proposed parallel algorithms and their respective serial versions.

5.2. Parallel Computing Platforms. The parallel computing architectures used in experiments are the Thunderhead
Table 1: Properties of the thermal hot spots reported in the rightmost part of Figure 12.

\begin{tabular}{|c|c|c|c|c|}
\hline $\begin{array}{l}\text { Hot } \\
\text { spot }\end{array}$ & $\begin{array}{l}\text { Latitude } \\
\text { (North) }\end{array}$ & $\begin{array}{l}\text { Longitude } \\
\text { (West) }\end{array}$ & $\begin{array}{c}\text { Temperature } \\
\text { (Kelvin) }\end{array}$ & $\begin{array}{c}\text { Area } \\
\text { (Square meters) }\end{array}$ \\
\hline "A" & $40^{\circ} 42^{\prime} 47.18^{\prime \prime}$ & $74^{\circ} 00^{\prime} 41.43^{\prime \prime}$ & 1000 & 0.56 \\
\hline "B" & $40^{\circ} 42^{\prime} 47.14^{\prime \prime}$ & $74^{\circ} 00^{\prime} 43.53^{\prime \prime}$ & 830 & 0.08 \\
\hline “C” & $40^{\circ} 42^{\prime} 42.89^{\prime \prime}$ & $74^{\circ} 00^{\prime} 48.88^{\prime \prime}$ & 900 & 0.80 \\
\hline “D” & $40^{\circ} 42^{\prime} 41.99^{\prime \prime}$ & $74^{\circ} 00^{\prime} 46.94^{\prime \prime}$ & 790 & 0.80 \\
\hline “E” & $40^{\circ} 42^{\prime} 40.58^{\prime \prime}$ & $74^{\circ} 00^{\prime} 50.15^{\prime \prime}$ & 710 & 0.40 \\
\hline "F" & $40^{\circ} 42^{\prime} 38.74^{\prime \prime}$ & $74^{\circ} 00^{\prime} 46.70^{\prime \prime}$ & 700 & 0.40 \\
\hline “G” & $40^{\circ} 42^{\prime} 39.94^{\prime \prime}$ & $74^{\circ} 00^{\prime} 45.37^{\prime \prime}$ & 1020 & 0.04 \\
\hline “H” & $40^{\circ} 42^{\prime} 38.60^{\prime \prime}$ & $74^{\circ} 00^{\prime} 43.51^{\prime \prime}$ & 820 & 0.08 \\
\hline
\end{tabular}


Beowulf cluster at NASA's Goddard Space Flight Center (NASA/GSFC) and a NVidia GeForce 9800 GX2 GPU.

(i) The Thunderhead Beowulf cluster is composed of $2.4 \mathrm{GHz}$ Intel Xeon nodes, each with $1 \mathrm{~GB}$ of memory and a scratch area of $80 \mathrm{~GB}$ of memory shared among the different processors (http://newton.gsfc.nasa.gov/thunderhead/). The total peak performance of the system is 2457.6 Gflops. Along with the 256-processor computer core (out of which only 32 were available to us at the time of experiments), Thunderhead has several nodes attached to the core with $2 \mathrm{GHz}$ optical fibre Myrinet [27]. The parallel algorithms tested in this work were run from one of such nodes, called thunder1 (used as the master processor in our tests). The operating system used at the time of experiments was Linux RedHat 8.0, and MPICH was the message-passing library used (http.//www.mcs.anl.gov/research/projects/mpi/mpich1). Figure 13(a) shows a picture of the Thunderhead Beowulf cluster.

(ii) The NVidia GeForce 9800 GX2 GPU contains two G92 graphics processors, each with 128 individual scalar processor (SP) cores and $512 \mathrm{MB}$ of fast DDR3 memory (http://www.nvidia.com/ object/product_geforce_9800gx2_us.html). The SPs are clocked at $1.5 \mathrm{GHz}$, and each can perform a fused multiply-add every clock cycle, which gives the card a theoretical peak performance of $768 \mathrm{GFlop} / \mathrm{s}$. The GPU is connected to a CPU Intel Q9450 with 4 cores, which uses a motherboard ASUS Striker II NSE (with NVidia 790i chipset) and $4 \mathrm{~GB}$ of RAM memory at $1333 \mathrm{MHz}$. Hyperspectral data are moved to and from the host CPU memory by DMA transfers over a PCI Express bus. Figure 13(b) shows a picture of the GeForce 9800 GX2 GPU.

5.3. Analysis of Target Detection Accuracy. It is first important to emphasize that our parallel versions of ATDCA and RX (implemented both for clusters and GPUs) provide exactly the same results as the serial versions of the same algorithms, implemented using the Intel $\mathrm{C} / \mathrm{C}++$ compiler and optimized via compilation flags to exploit data locality and avoid redundant computations. As a result, in order to refer to the target and anomaly detection results provided by the parallel versions of ATDCA and RX algorithms, we will refer to them as PG-ATDCA and PG-RX in order to indicate that the same results were achieved by the MPI-based and CUDA-based implementations for clusters and GPUs, respectively. At the same time, these results were also exactly the same as those achieved by the serial implementation and, hence, the only difference between the considered algorithms (serial and parallel) is the time they need to complete their calculations, which varies depending on the computer architecture in which they are run.

Table 2 shows the spectral angle distance (SAD) values (in degrees) between the most similar target pixels detected by PG-RX and PG-ATDCA (implemented using different distance metrics) and the pixel vectors at the known target positions, labeled from "A" to " $H$ " in the rightmost part of Figure 12. The lower the SAD score, the more similar the spectral signatures associated to the targets. In all cases, the number of target pixels to be detected was set to $t=30$ after calculating the virtual dimensionality (VD) of the data [34]. As shown by Table 2, both the PG-ATDCA and PGRX extracted targets were similar, spectrally, to the known ground-truth targets. The PG-RX was able to perfectly detect ( $\mathrm{SAD}$ of 0 degrees, represented in the table as $0^{\circ}$ ) the targets labeled as "A," "C," and "D" (all of them relatively large in size and with high temperature), while the PG-ATDCA implemented using OSP was able to perfectly detect the targets labeled as "C" and "D." Both the PG-RX and PGATDCA had more difficulties in detecting very small targets.

In the case of the PG-ATDCA implemented with a distance measure other than OSP we realized that, in many cases, some of the target pixels obtained were repeated. To solve this issue, we developed a method called relaxed pixel method (RPM) which simply removes a detected target pixel from the scene so that it cannot be selected in subsequent iterations. Table 3 shows the SAD between the most similar target pixels detected by P-ATDCA (implemented using the aforementioned RPM strategy) and the pixel vectors at the known target positions. It should be noted that the OSP distance implements the RPM strategy by definition and, hence, the results reported for PG-ATDCA in Table 3 are the same as those reported in Table 2 in which the RPM strategy is not considered. As shown by Table 3, most measured SADbased scores (in degrees) are lower when the RPM strategy is used, in particular, for targets of moderate size such as "A," "E," or "F." The detection results were also improved for the target with highest temperature, that is, the one labeled as "G." This indicated that the proposed RPM strategy can improve the detection results despite its apparent simplicity.

Finally, Table 4 shows a summary of the detection results obtained by the PG-RX and PG-ATDCA (with and without RPM strategy). It should be noted that it was not necessary to apply the RPM strategy to the PG-RX algorithm since this algorithm selects the final targets according to their value of $\delta^{(\mathrm{RXF})}(\mathbf{x})$ (the first pixel selected is the one with higher value of the RXF, then the one with the second higher value of the RXF, and so on). Hence, repetitions of targets are not possible in this case. In the table, the column "detected" lists those targets that were exactly identified (at the same spatial coordinates) with regards to the ground-truth, resulting in SAD value of exactly $0^{\circ}$ when comparing the associated spectral signatures. On the other hand, the column "similar" lists those targets that were identified with a SAD value below $30^{\circ}$ (a reasonable spectral similarity threshold taking in mind the great complexity of the scene, which comprises many different spectral classes). As shown by Table 4, the RPM strategy generally improved the results provided by the PGATDCA algorithm, both in terms of the number of detected targets and also in terms of the number of similar targets, in particular, when the algorithm was implemented using the SAD and SID distances. 


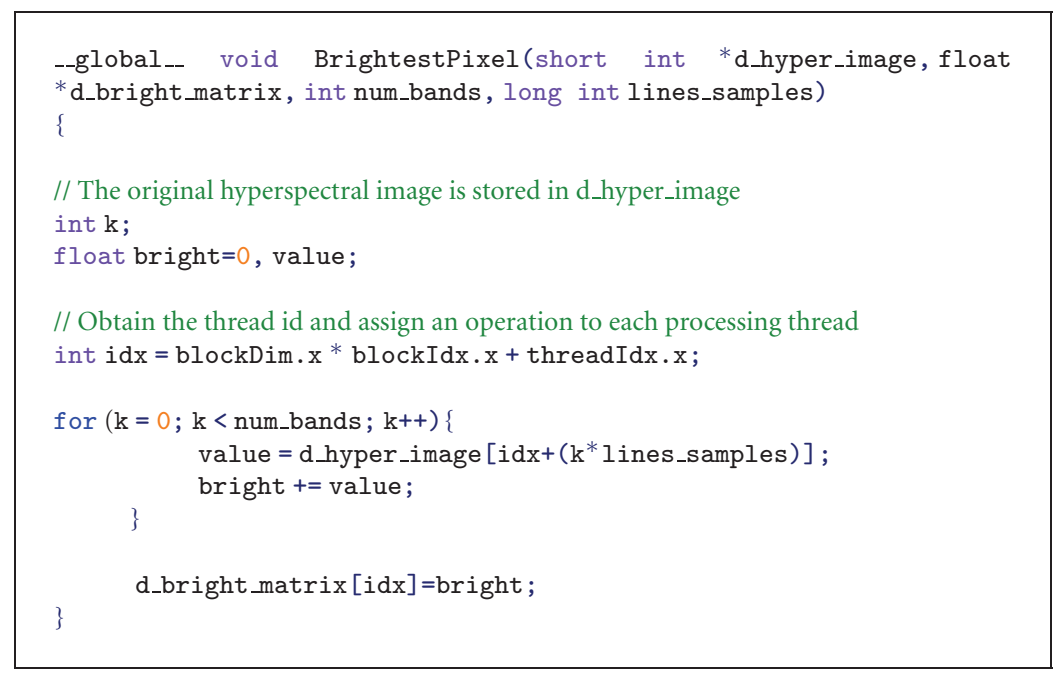

FIGURE 9: CUDA kernel BrightestPixel that computes (in parallel) the brightest pixel in the scene in the G-ATDCA implementation.

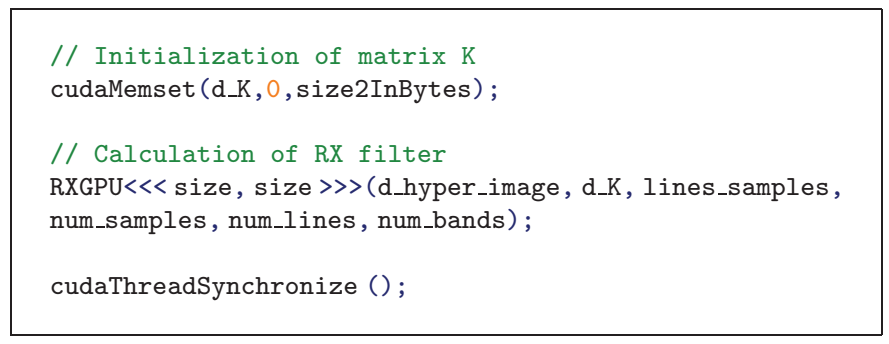

FIGURE 10: Portion of code which calls the CUDA kernel RXGPU designed to calculate the RX filter (in parallel) in the G-RX implementation.

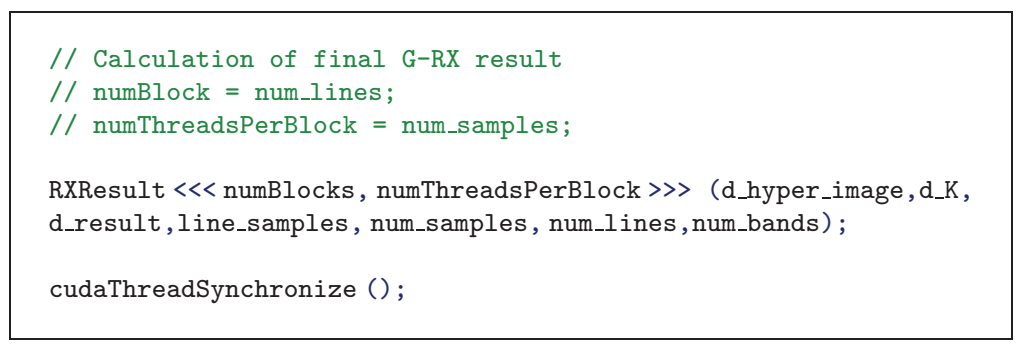

FIgUre 11: Portion of code which calls the CUDA kernel RXResult designed to obtain a final set of targets (in parallel) in the G-RX implementation.

TABLE 2: Spectral angle values (in degrees) between target pixels and known ground targets for PG-ATDCA and PG-RX.

\begin{tabular}{lcccccccc}
\hline Algorithm & A & B & C & D & E & F & G \\
\hline PG-ATDCA (OSP) & $9,17^{\circ}$ & $13,75^{\circ}$ & $0,00^{\circ}$ & $0,00^{\circ}$ & $20,05^{\circ}$ & $28,07^{\circ}$ & $21,20^{\circ}$ & $21,77^{\circ}$ \\
PG-ATDCA (1-Norm) & $9,17^{\circ}$ & $24,06^{\circ}$ & $0,00^{\circ}$ & $16,04^{\circ}$ & $37,82^{\circ}$ & $42,97^{\circ}$ & $38,39^{\circ}$ & $35,52^{\circ}$ \\
PG-ATDCA (2-Norm) & $9,17^{\circ}$ & $24,06^{\circ}$ & $0,00^{\circ}$ & $16,04^{\circ}$ & $37,82^{\circ}$ & $42,97^{\circ}$ & $38,39^{\circ}$ & $25,78^{\circ}$ \\
PG-ATDCA ( $\infty$-Norm) & $8,59^{\circ}$ & $22,35^{\circ}$ & $0,00^{\circ}$ & $13,75^{\circ}$ & $27,50^{\circ}$ & $30,94^{\circ}$ & $21,20^{\circ}$ & $26,36^{\circ}$ \\
PG-ATDCA (SAD) & $9,17^{\circ}$ & $22,35^{\circ}$ & $0,00^{\circ}$ & $14,32^{\circ}$ & $38,39^{\circ}$ & $32,09^{\circ}$ & $25,21^{\circ}$ & $29,79^{\circ}$ \\
PG-ATDCA (SID) & $9,17^{\circ}$ & $24,06^{\circ}$ & $0,00^{\circ}$ & $16,04^{\circ}$ & $39,53^{\circ}$ & $32,09^{\circ}$ & $22,92^{\circ}$ & $20,05^{\circ}$ \\
\hline PG-RX & $0,00^{\circ}$ & $12,03^{\circ}$ & $0,00^{\circ}$ & $0,00^{\circ}$ & $18,91^{\circ}$ & $28,07^{\circ}$ & $33,80^{\circ}$ & $40,68^{\circ}$ \\
\hline
\end{tabular}



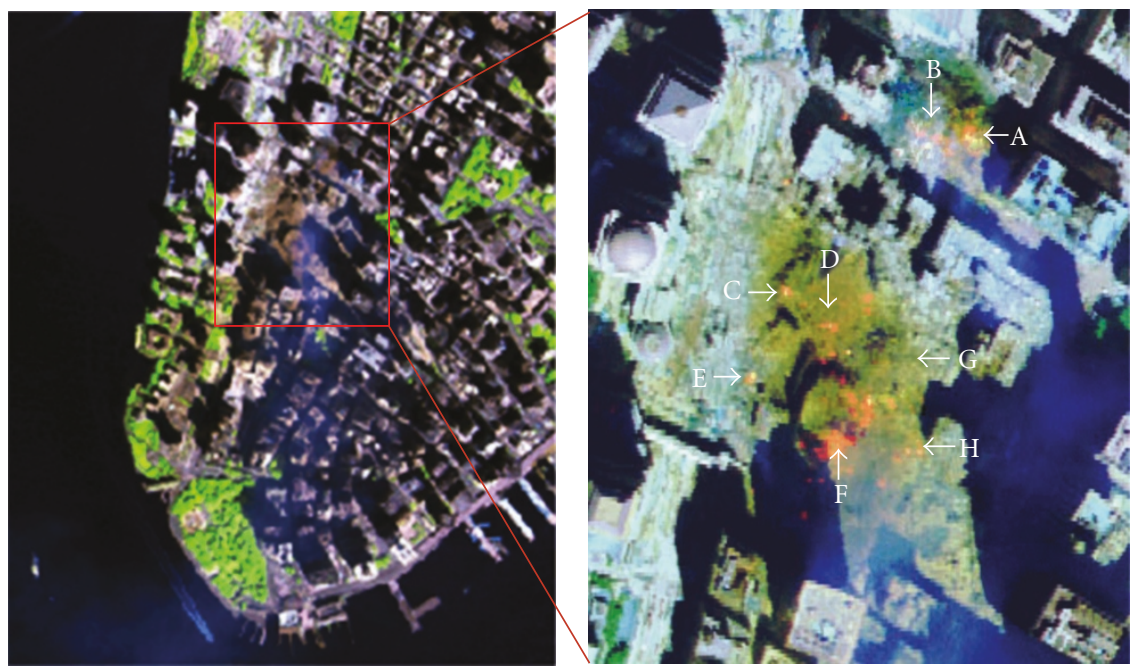

FIgURE 12: False color composition of an AVIRIS hyperspectral image collected by NASA's Jet Propulsion Laboratory over lower Manhattan on September 16, 2001 (left). Location of thermal hot spots in the fires observed in World Trade Center area, available online: http://pubs.usgs.gov/of/2001/ofr-01-0429/hotspot.key.tgif.gif (right).

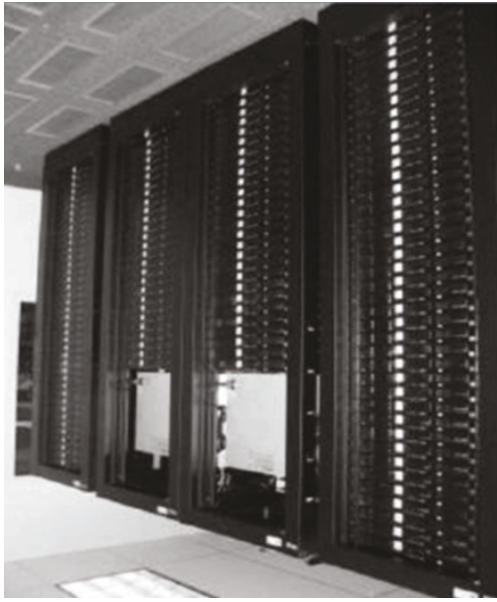

(a) Beowulf cluster

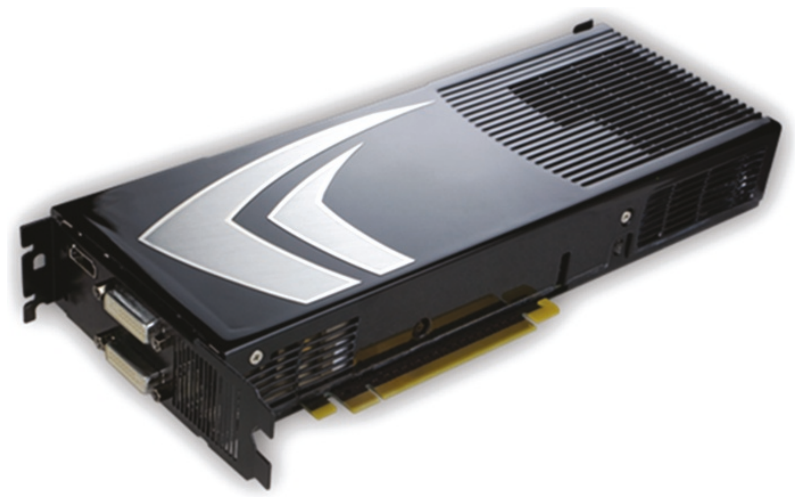

(b) GPU

Figure 13: (a) Thunderhead Beowulf cluster at NASA's Goddard Space Flight Center in Maryland. (b) NVidia GeForce 9800 GX2 GPU.

TABLE 3: Spectral angle values (in degrees) between target pixels and known ground targets for PG-ATDCA (implemented using RPM). The results reported for the OSP distance in PG-ATDCA are the same as those reported for the same distance in Table 2 since OSP implements the RPM strategy by definition.

\begin{tabular}{|c|c|c|c|c|c|c|c|c|}
\hline Algorithm & A & $\mathrm{B}$ & $\mathrm{C}$ & $\mathrm{D}$ & $\mathrm{E}$ & $\mathrm{F}$ & G & $\mathrm{H}$ \\
\hline PG-ATDCA (OSP) & $9,17^{\circ}$ & $13,75^{\circ}$ & $0,00^{\circ}$ & $0,00^{\circ}$ & $20,05^{\circ}$ & $28,07^{\circ}$ & $21,20^{\circ}$ & $21,77^{\circ}$ \\
\hline PG-ATDCA (1-Norm) & $0,00^{\circ}$ & $12,03^{\circ}$ & $0,00^{\circ}$ & $10,89^{\circ}$ & $22,35^{\circ}$ & $31,51^{\circ}$ & $34,38^{\circ}$ & $30,94^{\circ}$ \\
\hline PG-ATDCA (2-Norm) & $0,00^{\circ}$ & $14,90^{\circ}$ & $0,00^{\circ}$ & $10,89^{\circ}$ & $27,50^{\circ}$ & $31,51^{\circ}$ & $34,95^{\circ}$ & $25,78^{\circ}$ \\
\hline PG-ATDCA ( $\infty$-Norm) & $8,59^{\circ}$ & $14,90^{\circ}$ & $0,00^{\circ}$ & $13,75^{\circ}$ & $25,78^{\circ}$ & $30,94^{\circ}$ & $20,05^{\circ}$ & $26,36^{\circ}$ \\
\hline PG-ATDCA (SAD) & $0,00^{\circ}$ & $14,90^{\circ}$ & $0,00^{\circ}$ & $11,46^{\circ}$ & $29,79^{\circ}$ & $28,07^{\circ}$ & $22,92^{\circ}$ & $29,79^{\circ}$ \\
\hline PG-ATDCA (SID) & $0,00^{\circ}$ & $17,19^{\circ}$ & $0,00^{\circ}$ & $10,89^{\circ}$ & $30,94^{\circ}$ & $28,07^{\circ}$ & $22,35^{\circ}$ & $21,77^{\circ}$ \\
\hline
\end{tabular}


5.4. Parallel Performance in the Thunderhead Cluster. In this subsection we evaluate the parallel performance of both P-ATDCA and P-RX in a Beowulf cluster. Table 5 shows the processing times in seconds for several multiprocessor versions of P-RX and P-ATDCA using different numbers of processors (CPUs) on the Thunderhead Beowulf cluster at NASA's Goddard Space Flight Center. As shown by Table 5, when 32 processors were used, the P-ATDCA (implemented using SAD) was able to finalize in about 19 seconds, thus clearly outperforming the sequential version which takes 4 minutes of computation in one Thunderhead processor. In the case of P-RX, two versions were implemented: using communications when needed (communicative) and using redundant computations to reduce communications (independent), obtaining similar results in both cases. Here, the processing time using $32 \mathrm{CPUs}$ was only about 4 seconds, while the sequential time measured in one $\mathrm{CPU}$ was above one minute.

Table 6 reports the speedups (number of times that the parallel version was faster than the sequential one as the number of processors was increased) achieved by multiprocessor runs of the P-ATDCA algorithm (implemented using different distances) and P-RX. It can be seen that P-ATDCA (implemented using OSP and SID) scaled better than the two considered versions of P-RX. This has to do with the number of sequential computations involved in P-RX, as indicated in Figure 7. Another reason is the fact that, although the sample covariance matrix $\mathbf{K}$ required by this algorithm is calculated in parallel, its inverse is calculated serially at each node. In this regard, we believe that the speedups reported for the different implementations of P-RX in Table 6 could be improved even more if not only the calculation of the sample covariance matrix but also the inverse had been computed in parallel.

For illustrative purposes, the speedups achieved by the different implementations of P-ATDCA and P-RX are graphically illustrated in Figure 14. The speedup plots in Figure 14(a) reveal that P-ATDCA scaled better when OSP and SID were used as baseline distance metrics for implementation, resulting in speedups close to linear (although these distance measures introduced higher processing times, as indicated by Table 5). On the other hand, Figure 14(b) reveals that both versions of P-RX resulted in speedup plots that started to flatten from linear speedup from 16 CPUs in advance. This is probably due to the fact that the ratio of communications to computations increases as the partition size is made very small, an effect that is motivated by the high number of communications required by $\mathrm{P}-\mathrm{RX}$ as indicated by Figure 7.

Finally, Table 7 shows the load balancing scores for all considered parallel algorithms. The imbalance is defined as $D=\operatorname{Max} /$ Min, where Max and Min are the maxima and minima processor run times, respectively. Therefore, perfect balance is achieved when $D=1$. As we can see from Table 7, all the considered parallel algorithms were able to provide values of $D$ very close to optimal in the considered cluster, indicating that our implementations of P-ATDCA and P-RX achieved highly satisfactory load balance in all cases.
5.5. Parallel Performance in the GeForce 9800 GX2 GPU. In this subsection we evaluate the parallel performance of both G-ATDCA and G-RX in the NVidia GeForce 9800 GX2 GPU. Table 8 shows the execution times measured after processing the full hyperspectral scene $(614 \times 512$ pixels and 224 spectral bands) on the CPU and on the GPU, along with the speedup achieved in each case. The $\mathrm{C}$ function clock () was used for timing the CPU implementation, and the CUDA timer was used for the GPU implementation. The time measurement was started right after the hyperspectral image file was read to the CPU memory and stopped right after the results of the target/anomaly detection algorithm were obtained and stored in the CPU memory.

From Table 8, it can be seen that the G-ATDCA implemented using the OSP distance scaled slightly worse than the other implementations. This suggests that the matrix inverse and transpose operations implemented by the $P_{\mathrm{U}}^{\perp}$ orthogonal projection operator can still be optimized for efficient execution in the GPU. In this case, the speedup achieved by the GPU implementation over the optimized CPU implementation was only 3,4. When the G-ATDCA was implemented using the 1-Norm, 2-Norm, and $\infty$-Norm distances, the speedup increased to values around 10, for a total processing time below 10 seconds in the considered GPU. This can be defined as a significant accomplishment if we take in mind that just one GPU is used to parallelize the algorithm (in order to achieve similar speedups in the Thunderhead cluster, at least 16 CPUs were required). Table 8 also reveals that the speedups achieved by the GPU implementation were slightly increased when the SAD distance was used to implement the G-ATDCA. This suggests that the spectral angle calculations required for this distance can be efficiently parallelized in the considered GPU (in particular, calculation of cosines in the GPU was very efficient).

It is also clear from Table 8 that the best speedup results were obtained when the SID distance was used to implement the G-ATDCA. Specifically, we measured a speedup of 71,91 when comparing the processing time measured in the GPU with the processing time measured in the CPU. This is mainly due to the fact that the logarithm operations required to implement the SID distance can be executed very effectively in the GPU. Although the speedup achieved in this case is no less than impressive, the final processing time for the G-ATDCA implemented using this distance is still above two minutes after parallelization, which indicates that the use of the SID distance introduces additional complexity in both the serial and parallel implementations of the ATDCA algorithm. Similar comments apply to the parallel version of G-RX, which also takes more than 2 minutes to complete its calculations after parallelization. This is due to swapping problems in both of the serial implementation (i.e., an excessive traffic between disk pages and memory pages was observed, probably resulting from an ineffective allocation of resources in our G-RX implementation). This aspect should be improved in future developments of the parallel G-RX algorithm.

Summarizing, the experiments reported on Table 8 indicate that the considered GPU can significantly increase 
TABLE 4: Summary of detection results achieved by the PG-ATDCA and PG-RX, with and without the RPM strategy.

\begin{tabular}{lcccc}
\hline Algorithm & Detected & Similar & Detected (RPM) & Similar (RPM) \\
\hline PG-ATDCA (OSP) & C, D & A, B, E, F, G & C, D & A, B, E, F, G \\
PG-ATDCA (1-Norm) & C & A, B, D & A, C & B, D, E \\
PG-ATDCA (2-Norm) & C & A, B, D, H & A, C & B, D, E, H \\
PG-ATDCA ( $\infty$-Norm) & C & A, B, D, E, G, H & C & A, B, D, E, G, H \\
PG-ATDCA (SAD) & C & A, B, D, G, H & A, C & B, D, E, F, G, H \\
PG-ATDCA (SID) & C & A, B, D, G, H & A, C & B, D, F, G, H \\
\hline PG-RX & A, C, D & B, E, F & - & - \\
\hline
\end{tabular}

TABle 5: Processing times in seconds measured for P-ATDCA (implemented using different distance measures) and P-RX, using different numbers of CPUs on the Thunderhead Beowulf cluster.

\begin{tabular}{|c|c|c|c|c|c|c|}
\hline Algorithm & $1 \mathrm{CPU}$ & 2 CPUs & 4 CPUs & $8 \mathrm{CPUs}$ & 16 CPUs & $32 \mathrm{CPUs}$ \\
\hline P-ATDCA (OSP) & 1263,21 & 879,06 & 447,47 & 180,94 & 97,90 & 49,54 \\
\hline P-ATDCA (1-Norm) & 260,43 & 191,33 & 97,28 & 50,00 & 27,72 & 19,80 \\
\hline P-ATDCA (2-Norm) & 235,78 & 182,74 & 94,38 & 49,42 & 25,465 & 19,283 \\
\hline P-ATDCA $(\infty$-Norm $)$ & 268,95 & 187,92 & 99,28 & 50,96 & 27,75 & 22,00 \\
\hline P-ATDCA (SAD) & 241,93 & 187,83 & 96,14 & 49,24 & 25,35 & 19,00 \\
\hline P-ATDCA (SID) & 2267,60 & 1148,80 & 579,51 & 305,32 & 165,46 & 99,37 \\
\hline P-RX (Communicative) & 68,86 & 32,46 & 16,88 & 9,14 & 5,67 & 4,67 \\
\hline P-RX (Independent) & 68,86 & 32,70 & 16,82 & 8,98 & 5,46 & 4,42 \\
\hline
\end{tabular}

the performance of the considered algorithms, providing speedup ratios on the order of 10 for G-ATDCA (for most of the considered distances) and on the order of 14 for G-RX, although this algorithm should still be further optimized for more efficient execution on GPUs. When GATDCA was implemented using OSP as a baseline distance, the speedup decreased since the parallel matrix inverse and transpose operations are not fully optimized in our GPU implementation. On the other hand, when G-ATDCA was implemented using SID as a baseline distance, the speedup boosted over 71 due to the optimized capability of the considered GPU to compute logarithm-type operations in parallel. Overall, the best processing times achieved in experiments were on the order of 9 seconds. These response times are not strictly in realtime since the cross-track line scan time in AVIRIS, a push-broom instrument [3], is quite fast (8.3 milliseconds). This introduces the need to process the full image cube (614 lines, each made up of 512 pixels with 224 spectral bands) in about 5 seconds to achieve fully achieve real-time performance. Although the proposed implementations can still be optimized, Table 8 indicates that significant speedups can be obtained in most cases using only one GPU device, with very few on-board restrictions in terms of cost, power consumption, and size, which are important when defining mission payload (defined as the maximum load allowed in the airborne or satellite platform that carries the imaging instrument).

5.6. Discussion. In the previous subsections we have reported performance data for parallel target and anomaly detection algorithms implemented on a Beowulf cluster and a GPU.
From the obtained results, a set of remarks regarding the use of clusters versus GPUs for parallel processing of remotely sensed hyperspectral scenes follow.

5.6.1. Payload Requirements. A cluster of computers occupies much more space than a GPU, even if the PCs that form the cluster are concentrated in a compute core. If the cluster system is distributed across different locations, the space requirements increase. This aspect significantly limits the exploitation of cluster-based systems in on-board processing scenarios in the context of remote sensing, in which the weight of processing hardware must be limited in order to satisfy mission payload requirements. For example, a massively parallel cluster such as the Thunderhead system used in experiments occupies an area of several square meters with a total weight of several tons, requiring heavy cooling systems, uninterruptible power supplies, and so forth (see Figure 13(a)) In contrast, the GPU has the size of a PC card (see Figure 13(b)) and its weight is much more adequate in terms of current mission payload requirements. Most importantly, our experimental results have indicated that using just one GPU we can obtain parallel performance results which are equivalent to those obtained using tens of nodes in a cluster, thus significantly reducing the weight and space occupied by hardware resources while maintaining the same parallel performance.

5.6.2. Maintenance. The maintenance of a large cluster represents a major investment in terms of time and finance. Each node of the cluster is a computer in itself, with its own operating system, possible deterioration of components, 


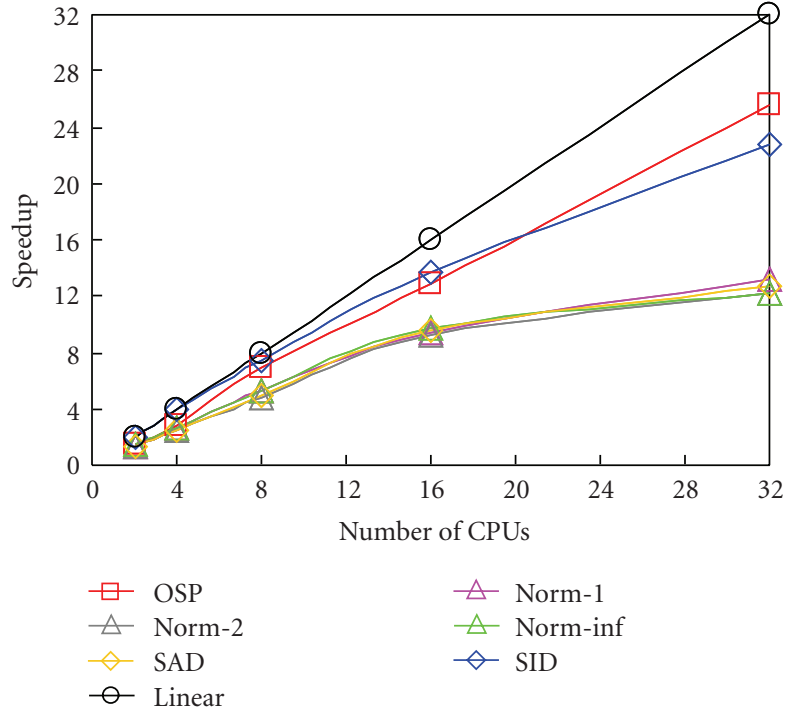

(a) P-ATDCA

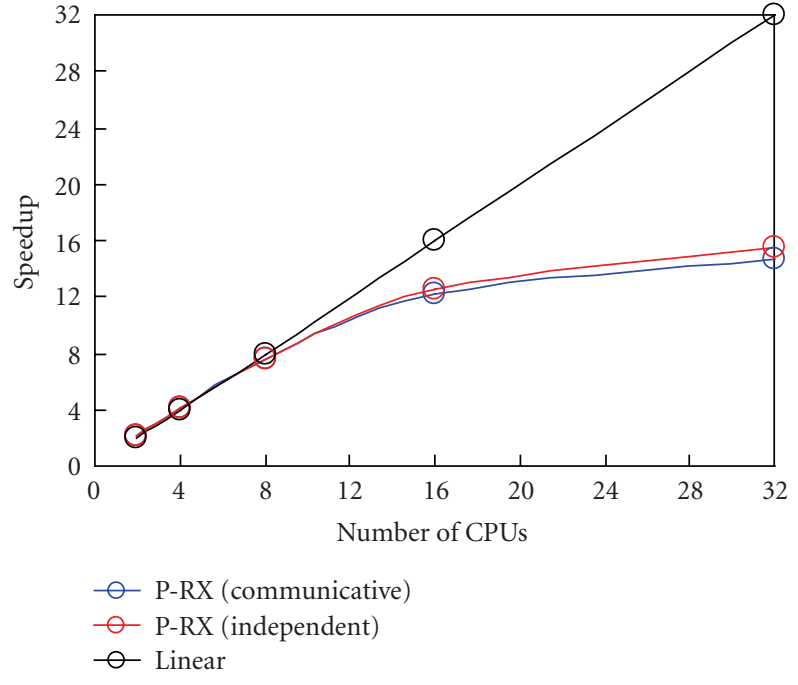

(b) P-RX

FIGURE 14: Speedups achieved by (a) P-ATDCA (using different distance measures) and (b) P-RX (communicative and independent versions) on the Thunderhead Beowulf cluster at NASA's Goddard Space Flight Center in Maryland.

TABle 6: Speedups for the P-ATDCA algorithm (using different distance measures) and P-RX using different numbers of CPUs on the Thunderhead Beowulf cluster.

\begin{tabular}{|c|c|c|c|c|c|}
\hline Algorithm & 2 CPUs & 4 CPUs & 8 CPUs & 16 CPUs & 32 CPUs \\
\hline P-ATDCA (OSP) & 1,437 & 2,823 & 6,981 & 12,902 & 25,498 \\
\hline P-ATDCA (1-Norm) & 1,386 & 2,677 & 5,208 & 9,393 & 13,148 \\
\hline P-ATDCA (2-Norm) & 1,290 & 2,498 & 4,770 & 9,258 & 12,227 \\
\hline P-ATDCA $(\infty$-Norm $)$ & 1,431 & 2,708 & 5,277 & 9,690 & 12,224 \\
\hline P-ATDCA (SAD) & 1,288 & 2,516 & 4,913 & 9,542 & 12,727 \\
\hline P-ATDCA (SID) & 1,973 & 3,912 & 7,426 & 13,704 & 22,818 \\
\hline P-RX (Communicative) & 2,121 & 4,079 & 7,531 & 12,140 & 14,720 \\
\hline P-RX (Independent) & 2,105 & 4,092 & 7,662 & 12,594 & 15,558 \\
\hline
\end{tabular}

system failures, and so forth. This generally requires a team of dedicated system administrators, depending on the size of the cluster, to ensure that all the nodes in the system are running. In general terms, the maintenance costs for a cluster with $P$ processing nodes is similar to the maintenance costs for $P$ independent machines. However, the maintenance cost for a GPU is similar to that of the administration cost of a single machine. As a result, the advantages of a GPU with regards to a cluster from the viewpoint of the maintenance of the system are quite important, in particular, in the context of remote sensing data analysis scenarios in which compact hardware devices, which can be mounted onboard imaging instruments, are highly desirable. Regarding possible hardware failures in both systems, it is worth noting that such failures are generally easier to manage in GPUbased systems rather than in cluster systems, in which the failure may require several operations such as identifying the node that caused the failure, removing the node, finding out which software/hardware components caused the error, repairing/changing the defective components, reinstall the software (if necessary), and reconnecting it.

5.6.3. Cost. Although a cluster is a relatively inexpensive parallel architecture, the cost of a cluster can increase significantly with the number of nodes. The estimated cost of a system such as Thunderhead, assuming a conservative estimate of 600 USD per each node, is in the order of $600 \times 256=153,600$ USD (without including the cost of the communication network). In turn, the cost of a relatively modern GPU such as the GeForce 9800 GX2 GT used in our experiments is now below 500 USD. Our experiments reveal that the parallel performance obtained in the GPU can be superior to that obtained using 32 nodes of the Thunderhead system, and the cost of such nodes (without including the cost of the communication network) is around $32 \times 256=8,192$ USD. This reveals the important advantages introduced by GPUs in the sense of providing high-performance computing at lower costs 
TABle 7: Load balancing ratios for the P-ATDCA (implemented using different distance measures) and P-RX (communicative and independent versions).

\begin{tabular}{|c|c|c|c|c|c|c|}
\hline Algorithm & Imbalance & 2 CPUs & 4 CPUs & 8 CPUs & 16 CPUs & 32 CPUs \\
\hline \multirow{3}{*}{ P-ATDCA (OSP) } & $\operatorname{Max}$ & 879,06 & 447,47 & 180,94 & 97,90 & 49,54 \\
\hline & Min & 878,94 & 447,01 & 180,23 & 97,06 & 48,95 \\
\hline & $D$ & 1,00013652 & 1,0010290 & 1,00393941 & 1,00865444 & 1,0120531 \\
\hline \multirow{3}{*}{ P-ATDCA (1-Norm) } & Max & 191,33 & 97,28 & 50,00 & 27,74 & 19,81 \\
\hline & Min & 191,32 & 97,27 & 49,98 & 27,72 & 19,80 \\
\hline & $D$ & 1,00005227 & 1,0001028 & 1,00030008 & 1,00072137 & 1,00055536 \\
\hline \multirow{3}{*}{ P-ATDCA (2-Norm) } & Max & 182,75 & 94,38 & 49,42 & 25,47 & 19,29 \\
\hline & Min & 182,74 & 94,37 & 49,41 & 25,46 & 19,28 \\
\hline & $D$ & 1,00005472 & 1,00006357 & 1,00020235 & 1,00047124 & 1,00072603 \\
\hline \multirow{3}{*}{ P-ATDCA ( $\infty$-Norm) } & Max & 187,93 & 99,29 & 50,97 & 27,77 & 22,01 \\
\hline & Min & 187,92 & 99,28 & 50,96 & 27,75 & 22,00 \\
\hline & $D$ & 1,00005321 & 1,0000705 & 1,00019623 & 1,0006125 & 1,00068179 \\
\hline \multirow{3}{*}{ P-ATDCA (SAD) } & Max & 187,83 & 96,14 & 49,24 & 25,35 & 19,01 \\
\hline & Min & 187,83 & 96,13 & 49,23 & 25,33 & 19,00 \\
\hline & $D$ & 1 & 1,00008321 & 1,00010155 & 1,00059202 & 1,00073669 \\
\hline \multirow{3}{*}{ P-ATDCA (SID) } & Max & 1148,80 & 579,52 & 305,33 & 165,47 & 99,39 \\
\hline & Min & 1148,80 & 579,51 & 305,32 & 165,46 & 99,375 \\
\hline & $D$ & 1 & 1,00001726 & 1,00003275 & 1,00006044 & 1,00016101 \\
\hline \multirow{3}{*}{ P-RX (Communicative) } & Max & 32,46 & 16,88 & 9,14 & 5,67 & 4,67 \\
\hline & Min & 32,46 & 16,79 & 8,92 & 5,50 & 4,5264 \\
\hline & $D$ & 1 & 1,00553901 & 1,02495096 & 1,03130568 & 1,03360286 \\
\hline \multirow{3}{*}{ P-RX (Independent) } & Max & 32,70 & 16,82 & 8,98 & 5,46 & 4,42 \\
\hline & Min & 32,47 & 16,68 & 8,95 & 5,46 & 4,41 \\
\hline & $D$ & 1,00701992 & 1,00869252 & 1,00334919 & 1,00131851 & 1,00194667 \\
\hline
\end{tabular}

TABle 8: Processing time (seconds) and speedups measured for the CPU and GPU implementations of several target and anomaly detection algorithms.

\begin{tabular}{lccc}
\hline Algorithm & $\begin{array}{c}\text { Processing time } \\
(\mathrm{CPU})\end{array}$ & $\begin{array}{c}\text { Processing time } \\
(\mathrm{GPU})\end{array}$ & Speedup \\
\hline G-ATDCA (OSP) & 1263,21 & 370,96 & 3,40 \\
G-ATDCA (1-Norm) & 99,24 & 9,03 & 10,98 \\
G-ATDCA (2-Norm) & 83,99 & 9,41 & 9,28 \\
G-ATDCA ( $\infty$-Norm) & 109,28 & 9,05 & 12,07 \\
G-ATDCA (SAD) & 133,63 & 9,06 & 14,74 \\
G-ATDCA (SID) & 911,85 & 12,67 & 71,91 \\
\hline G-RX & 1955,15 & 139,17 & 14,04 \\
\hline
\end{tabular}

than those generally observed for commodity cluster-based systems.

5.6.4. Memory Considerations. A cluster of $P$ nodes is a distributed memory system in which the $P$ processors have $P$ independent memory systems and $P$ copies of the operating system, each subject to local failures. Although a scratch disk area is usually allocated in parallel clusters for common use of the different processing nodes, the memory in these systems is distributed. However, the GPU is a sharedmemory system in which the local memory space is shared by all the multiprocessors in the GPU. This avoids the problems introduced by parallel algorithms with heavy interprocessor communications such as the P-RX illustrated in Figure 7 since these algorithms can be implemented by assuming that shared local memory will be available to all processing elements in the system, thus reducing quite significantly the penalties introduced by excessive communications while, at the same time, increasing the ratio of computations to communications. This generally results in better parallel performance, as observed in our experimental results.

From the observations above, we can conclude that commodity cluster-based parallel systems are indeed an appealing solution in order to process remote sensing image data sets which have been already transmitted to Earth. PC workstations are everywhere, and it is not difficult to put together a network and/or a cluster, given the raw materials. For instance, the processing power offered by such commodity systems has been traditionally employed in data mining applications from very large data archives, possibly distributed among different geographic locations. However, compact hardware devices such as GPUs offer significant advantages in time-critical applications that demand a response in real-time (i.e., at the same time as 
the data is collected at the sensor) mainly due to the low weight and size of these devices, and to their capacity to provide high performance computing at very low costs. In previous work [29], we have quantitatively compared the performance of clusters versus field programmable gate arrays (FPGAs) in the context of remote sensing applications. FPGAs are another type of compact hardware device that offer interesting perspectives in our application domain, such as the appealing possibility of being able to adaptively select the data processing algorithm to be applied (out of a pool of available algorithms) from a control station on Earth, immediately after the data is collected by the sensor. This feature is possible thanks to the inherent reconfigurability of FPGA devices, which are generally more expensive than GPU devices. In the future, significant developments are expected in the active research area devoted to radiationhardening of GPU and FPGA devices, which may allow their full incorporation to satellite-based Earth and planetary observation platforms in space. These systems represent the next frontier of hyperspectral remote sensing.

\section{Conclusions and Future Research}

With the ultimate goal of drawing a comparison of clusters versus GPUs as high-performance computing architectures in the context of remote sensing applications, this paper described several innovative parallel algorithms for target and anomaly detection in hyperspectral image analysis. As a case study of specific issues involved in the exploitation of an automatic algorithm for target detection and classification (ATDCA), we have investigated the impact of including several distance measures in the design of different parallel versions of this algorithm. This paper has also developed a new parallel version of a well-known anomaly detection algorithm (RX). The parallel algorithms have been implemented in two types of parallel computing platforms: a Beowulf cluster at NASA's Goddard Space Flight Center in Maryland and an NVidia GeForce 9800 GX2 GPU. Experimental results, oriented towards analyzing the target/anomaly detection accuracy and parallel performance of the proposed parallel algorithms, have been presented and thoroughly discussed in the context of a real defense and security application: the analysis of hyperspectral data collected by NASA's AVIRIS instrument over the World Trade Center (WTC) in New York, five days after the terrorist attacks that collapsed the two main towers in the WTC complex. Our experimental assessment of clusters versus GPUs in the context of this particular application indicates that commodity clusters represent a source of computational power that is both accessible and applicable to obtaining results quickly enough and with high reliability in target/anomaly detection applications in which the data has already been transmitted to Earth. However, GPU hardware devices may offer important advantages in defense and security applications that demand a response in realtime, mainly due to the low weight and compact size of these devices, and to their capacity to provide high-performance computing at very low costs.
Although the results reported in this work are very encouraging, further experiments should be conducted in order to increase the parallel performance of the proposed parallel algorithms by resolving memory issues in the clusterbased implementations and optimizing the parallel design of the algorithms in the GPU-based implementations. Regarding the cluster-based implementation of the RX algorithm reported in this work, we are planning on implementing not only the sample covariance matrix but also the inverse in parallel in order to increase scalability in future developments. Experiments with additional scenes under different target/anomaly detection scenarios are also highly desirable. Finally, experiments with radiation-hardened GPU devices will be required in order to evaluate the possibility of adapting the proposed parallel algorithms to hardware devices which have been already certified by international agencies and are mounted on-board satellite platforms for Earth and planetary observation from space.

\section{Acknowledgments}

This work has been supported by the European Community's Marie Curie Research Training Networks Programme under reference MRTN-CT-2006-035927 (HYPER-I-NET). Funding from the Spanish Ministry of Science and Innovation (HYPERCOMP/EODIX project, reference AYA2008-05965C04-02), is gratefully acknowledged.

\section{References}

[1] A. F. H. Goetz, G. Vane, J. E. Solomon, and B. N. Rock, "Imaging spectrometry for earth remote sensing.," Science, vol. 228, no. 4704, pp. 1147-1153, 1985.

[2] A. Plaza, J. A. Benediktsson, J. W. Boardman, et al., "Recent advances in techniques for hyperspectral image processing," Remote Sensing of Environment, vol. 113, supplement 1, pp. S110-S122, 2009.

[3] R. O. Green, M. L. Eastwood, C. M. Sarture, et al., "Imaging spectroscopy and the Airborne Visible/Infrared Imaging Spectrometer (AVIRIS)," Remote Sensing of Environment, vol. 65, no. 3, pp. 227-248, 1998.

[4] C.-I. Chang, Hyperspectral Imaging: Techniques for Spectral Detection and Classification, Kluwer Academic Publishers, Norwell, Mass, USA, 2003.

[5] A. Plaza and C.-I. Chang, High Performance Computing in Remote Sensing, CRC Press, Boca Raton, Fla, USA, 2007.

[6] R. A. Schowengerdt, Remote Sensing: Models and Methods for Image Processing, Academic Press, New York, NY, USA, 2nd edition, 1997.

[7] D. A. Landgrebe, Signal Theory Methods in Multispectral Remote Sensing, John Wiley \& Sons, New York, NY, USA, 2003.

[8] J. A. Richards and X. Jia, Remote Sensing Digital Image Analysis: An Introduction, Springer, London, UK, 2006.

[9] C.-I. Chang, Recent Advances in Hyperspectral Signal and Image Processing, John Wiley \& Sons, New York, NY, USA, 2007.

[10] C.-I. Chang, Hyperspectral Data Exploitation: Theory and Applications, John Wiley \& Sons, New York, NY, USA, 2007.

[11] C.-I. Chang and H. Ren, "An experiment-based quantitative and comparative analysis of target detection and image 
classification algorithms for hyperspectral imagery," IEEE Transactions on Geoscience and Remote Sensing, vol. 38, no. 2, pp. 1044-1063, 2000.

[12] H. Ren and C.-I. Chang, "Automatic spectral target recognition in hyperspectral imagery," IEEE Transactions on Aerospace and Electronic Systems, vol. 39, no. 4, pp. 1232-1249, 2003.

[13] D. Manolakis, D. Marden, and G. A. Shaw, "Hyperspectral image processing for automatic target detection applications," MIT Lincoln Laboratory Journal, vol. 14, pp. 79-116, 2003.

[14] A. Paz, A. Plaza, and S. Blazquez, "Parallel implementation of target and anomaly detection algorithms for hyperspectral imagery," in Proceedings of International Geoscience and Remote Sensing Symposium (IGARSS '08), vol. 2, pp. 589-592, 2008.

[15] Y. Tarabalka, T. V. Haavardsholm, I. Kasen, and T. Skauli, "Real-time anomaly detection in hyperspectral images using multivariate normal mixture models and GPU processing," Journal of Real-Time Image Processing, vol. 4, no. 3, pp. 287300, 2009.

[16] D. C. Heinz and C.-I. Chang, "Fully constrained least squares linear spectral mixture analysis method for material quantification in hyperspectral imagery," IEEE Transactions on Geoscience and Remote Sensing, vol. 39, no. 3, pp. 529-545, 2001.

[17] R. A. Neville, K. Staenz, T. Szeredi, J. Lefebvre, and P. Hauff, "Automatic endmember extraction from hyperspectral data for mineral exploration," in Proceedings of the 21st Canadian Symposium on Remote Sensing, pp. 401-415, 1999.

[18] I. S. Reed and X. Yu, "Adaptive multiple-band CFAR detection of an optical pattern with unknown spectral distribution," IEEE Transactions on Acoustics, Speech, and Signal Processing, vol. 38, no. 10, pp. 1760-1770, 1990.

[19] J. C. Harsanyi and C.-I. Chang, "Hyperspectral image classification and dimensionality reduction: an orthogonal subspace projection approach," IEEE Transactions on Geoscience and Remote Sensing, vol. 32, no. 4, pp. 779-785, 1994.

[20] N. Acito, M. Diani, and G. Corsini, "A new algorithm for robust estimation of the signal subspace in hyperspectral images in the presence of rare signal components," IEEE Transactions on Geoscience and Remote Sensing, vol. 47, no. 11, pp. 3844-3856, 2009.

[21] A. Plaza, P. Martinez, J. Plaza, and R. Perez, "Dimensionality reduction and classification of hyperspectral image data using sequences of extended morphological transformations," IEEE Transactions on Geoscience and Remote Sensing, vol. 43, no. 3, pp. 466-479, 2005.

[22] C.-I. Chang and D. C. Heinz, "Constrained subpixel target detection for remotely sensed imagery," IEEE Transactions on Geoscience and Remote Sensing, vol. 38, no. 3, pp. 1144-1159, 2000.

[23] N. Acito, G. Corsini, and M. Diani, "Computational load reduction for anomaly detection in hyperspectral images: an experimental comparative analysis," in Proceedings of International Geoscience and Remote Sensing Symposium (IGARSS '08), pp. 3206-3209, 2008.

[24] K. Itoh, "Massively parallel Fourier-transform spectral imaging and hyperspectral image processing," Optics and Laser Technology, vol. 25, p. 202, 1993.

[25] T. El-Ghazawi, S. Kaewpijit, and J. L. Moigne, "Parallel and adaptive reduction of hyperspectral data to intrinsic dimensionality," Cluster Computing, vol. 1, pp. 102-110, 2001.
[26] A. Plaza, J. Plaza, and D. Valencia, "Impact of platform heterogeneity on the design of parallel algorithms for morphological processing of high-dimensional image data," Journal of Supercomputing, vol. 40, no. 1, pp. 81-107, 2007.

[27] J. Dorband, J. Palencia, and U. Ranawake, "Commodity computing clusters at goddard space flight center," Journal of Space Communication, vol. 3, p. 1, 2003.

[28] A. Plaza, D. Valencia, J. Plaza, and P. Martinez, "Commodity cluster-based parallel processing of hyperspectral imagery," Journal of Parallel and Distributed Computing, vol. 66, no. 3, pp. 345-358, 2006.

[29] A. Plaza and C.-I. Chang, "Clusters versus FPGA for parallel processing of hyperspectral imagery," International Journal of High Performance Computing Applications, vol. 22, no. 4, pp. 366-385, 2008.

[30] J. Setoain, M. Prieto, C. Tenllado, A. Plaza, and F. Tirado, "Parallel morphological endmember extraction using commodity graphics hardware," IEEE Geoscience and Remote Sensing Letters, vol. 4, no. 3, pp. 441-445, 2007.

[31] A. Paz, A. Plaza, and J. Plaza, "Comparative analysis of different implementations of a parallel algorithm for automatic target detection and classification of hyperspectral images," in Satellite Data Compression, Communication, and Processing V, vol. 7455 of Proceedings of SPIE, San Diego, Calif, USA, August 2009.

[32] J. C. Tilton, W. T. Lawrence, and A. J. Plaza, "Utilizing hierarchical segmentation to generate water and snow masks to facilitate monitoring change with remotely sensed image data," GIScience and Remote Sensing, vol. 43, no. 1, pp. 39-66, 2006.

[33] R. Brightwell, L. A. Fisk, D. S. Greenberg, et al., "Massively parallel computing using commodity components," Parallel Computing, vol. 26, no. 2, pp. 243-266, 2000.

[34] C.-I. Chang and Q. Du, "Estimation of number of spectrally distinct signal sources in hyperspectral imagery," IEEE Transactions on Geoscience and Remote Sensing, vol. 42, no. 3, pp. 608-619, 2004. 